\title{
Error Vector Magnitude Analysis of Fading SIMO Channels Relying on MRC Reception
}

\author{
Varghese Antony Thomas, Suman Kumar, Sheetal Kalyani, Mohammed El-Hajjar, K. Giridhar, \\ and Lajos Hanzo, Fellow, IEEE
}

\begin{abstract}
5 Abstract-We analytically characterize the data-aided error 6 vector magnitude (EVM) performance of a single-input multiple7 output (SIMO) communication system relying on maximal ratio 8 combining (MRC) having either independent or correlated 9 branches that are nonidentically distributed. In particular, exact 10 closed form expressions are derived for the EVM in $\eta-\mu$ fading and $1 \kappa-\mu$ shadowed fading channels and these expressions are validated 12 by simulations. The derived expressions are expressed in terms 13 of Lauricella's function of the fourth kind $F_{D}^{(N)}($.$) , which can$ 14 be easily computed. Furthermore, we have simplified the derived 15 expressions for various special cases such as independent and iden16 tically distributed branches, Rayleigh fading, Nakagami- $m$ fading, 17 and $\kappa-\mu$ fading. Additionally, a parametric study of the EVM 18 performance of the wireless system is presented.
\end{abstract}

Index Terms-Error Vector Magnitude, maximal ratio combining, $\eta-\mu$ fading, $\kappa-\mu$ fading, SIMO.

\section{INTRODUCTION}

$\mathbf{C}$ ONFORMITY with the wireless communication performance standards is an absolute necessity, when designing communication systems. Traditional approaches of quantifying a communication system's performance includes the calculation of classic metrics such as the Bit Error Ratio (BER), the throughput and the outage probability [1]-[4]. However, an alternative metric that is becoming increasingly popular is the Error Vector Magnitude (EVM) [5].

EVM as a performance metric offers several advantages. Firstly, it facilitates the identification of the specific types of degradations encountered, in addition to their particular sources in a transmission link [5]. Some of these degradations are the Inphase-Quadrature Phase (IQ) imbalance, the Local Oscillator's (LO) phase noise, carrier leakage, nonlinearity and the LO's frequency error [6], [7]. Secondly, the EVM is a symbol-level performance metric unlike the BER, which is a

Manuscript received July 21, 2015; revised December 12015 and February 8, 2016; accepted February 12, 2016. The financial support of the EPSRC projects EP/Noo4558/1 and EP/L018659/1, as well as of the European Research Council's Advanced Fellow Grant under the Beam-Me-Up project and of the Royal Society's Wolfson Research Merit Award is gratefully acknowledged. V. A. Thomas and S. Kumar are co-first authors. The associate editor coordinating the review of this paper and approving it for publication was T. A. Tsiftsis.

V. A. Thomas, M. El-Hajjar, and L. Hanzo are with the School of Electronics and Computer Science, University of Southampton, Southampton SO17 1BJ, U.K. (e-mail: 1h@ecs.soton.ac.uk).

S. Kumar, S. Kalyani, and K. Giridhar are with the Department of Electrical Engineering, Indian Institute of Technology Madras, Chennai 600036, India.

Color versions of one or more of the figures in this paper are available online at http://ieeexplore.iee.org.

Digital Object Identifier 10.1109/TCOMM.2016.2530797 bit-level performance metric. Hence, EVM is more convenient for Symbol Error Rate (SER) based scenarios where multiple modulation schemes are employed, as in adaptive modulation [8]. Thirdly, it may be employed by a communication system designer for ensuring conformity with wireless standards, because EVM-based specifications have already become a part of the Wideband Code Division Multiple Access (W-CDMA) and IEEE 802.11 family of Wireless Local Area Network (WLAN) standards [5], [8]. Fourthly, in experimental studies the channel model used is often a proprietary channel, for which no closed form expressions are available either for the BER or for the EVM. In these studies, the designer has to characterize the system by transmitting and receiving bits, where the BER calculation relying on the Monte Carlo approach has a long computation time, especially at low BERs. By contrast, the EVM can be readily evaluated by transmitting fewer symbols, as compared to the BER. Hence, characterizing the performance using EVM is preferred. However, in contrast to the classic BER formulae, the current literature does not provide closed form expressions of the EVM of several important channel scenarios. Hence provides closed-form expressions for some of these important channel scenarios and partially fills this gap in the open literature. We have now added the following text to the discussions in the introduction section (please see page 2 of the revised manuscript). Moreover, EVM is easier to employ than BER as a performance metric in systems, where the transmitter requires feedback regarding the link's performance for making choices such as which adaptive modulation mode or channel coding rate to rely on. This is because employing BER would require the received signal to go through the entire receive chain before the feedback can be generated, while computation of the EVM using the received symbols would be quicker. Thus, employing EVM would be a better choice for providing real-time feedback.

In an optimized system the major source of degradation is the channel's fading [4], [9]. However, in a realistic system a range of degradations mentioned in [5] are imposed, which would play a detrimental role. Employing EVM would help the designer identify these impairments at a glance and hence to mitigate them. Mitigating the effects of these distortions would require the EVM of the the best-case scenario, where the EVM is predominantly or purely decided by the wireless channel's fading as well as by the ubiquitous receiver-noise, and not by other impairments, such as non-linear distortions and synchronization errors, etc. Hence in this paper we aim for providing the designer with closed form expressions for 
determining this best-case target EVM. Numerous models have been employed in the literature for simulating a wireless channel [4]. Some of these models have been used for several years, including the AWGN, Rayleigh, Rician and the Nakagami-m as well as the Nakagami-q faded channels. On the other hand, recent studies are increasingly favouring the state-of-the-art $\eta-\mu$ and $\kappa-\mu$ shadowed fading channels [10], [11], because they represent all-encompassing generalizations, with the classical channels being their special cases. For example, the $\eta-\mu$ distribution includes the Nakagami-q (Hoyt), the Nakagami-m, the Rayleigh and the One-Sided Gaussian distribution as special cases. The $\kappa-\mu$ distribution includes the Nakagami-n (Rice), the Nakagami-m, the Rayleigh, and the One-Sided Gaussian distribution as special cases. The $\kappa-\mu$ shadowed distribution includes $\kappa-\mu$ and Rician shadowed distribution as special cases. Moreover, they match the experimentally measured mobile radio propagation statistics better than the other channel models [10]. The $\kappa-\mu$ shadowed fading is useful for modelling the satellite links. A simplified model for $\kappa-\mu$ fading is the shadowed-Rician fading, which has been employed for modelling the satellite links [12]-[15].

The BER, outage probability and capacity are some commonly employed performance metrics, which have been quantified for $\eta-\mu$ and $\kappa-\mu$ shadowed fading ${ }^{1}$ channels in [16]-[19] and in the references therein. On the other hand, there is a dearth of studies that focus on the quantification of the achievable EVM for these wireless channels. Moreover, there are no studies that characterize the EVM performance for the commonly employed wireless technique of receive antenna diversity [20]. Note that a performance analysis of maximal ratio combining based receive antenna diversity was provided in [21] for the case of the shadowed-Rician fading land mobile satellite channels. Employing multiple receive antennas provides a diversity gain [20], where the link between the transmit antenna and each receive antenna is referred to as a single branch of the Single Input Multiple Output (SIMO) channel. The fading coefficients of the different branches may be independently distributed or correlated, where the branches in these scenearios are referred to as being independent or correlated, respectively. Additionally, they may have the same or different probability distribution parameters, where the branches in these scenearios are referred to as being identically or non-identically distributed, respectively. It must be noted that there is some literature on the EVM performance of the classical AWGN and Rayleigh channels for the scenario of a single receive antenna, though these are limited to only a couple of research papers. The seminal effort was made in this direction in [22], while [23] formulates the attainable EVM in an AWGN scenario. This study was extended in [24] to the scenario of non data-aided receivers communicating over both $\mathrm{AWGN}$ as well as Rayleigh fading channels.

A designer can compute the expected BER for various fading channels using well established formulae from the existing literature. Thus, designers have a benchmark with which they

\footnotetext{
${ }^{1}$ The probability distribution function (pdf) of the sum of the squared $\kappa-\mu$ shadowed random variables with independent and correlated shadowing components are derived in [11] and [16], respectively. Note that the pdf derived in [11] is a special case of the pdf derived in [16].
}

can compare the experimental results, when using BER as a 138 performance metric. However, there are no such equivalent the- 139 oretical formulae for EVM. Hence, through this paper we aim 140 to provide a theoretical benchmark for the EVM performance 141 that the designer can expect in the wireless channels. 142

Against this background, the novel contributions of this 143 paper may be summarised as follows:

1) We derive exact closed form expressions for the dataaided $\mathrm{EVM}^{2}$ performance of a SIMO wireless system employing the $\eta-\mu$ and $\kappa-\mu$ shadowed fading channels and a Maximal Ratio Combining (MRC) receiver. Our expressions are derived for independent and non identically distributed branches. These results are then validated by simulations ${ }^{3}$.

2) We also study the effect of correlated fading channels in the above-mentioned wireless system and formulate the EVM for these scenarios.

3) The expressions derived are then further simplified for 155 various special cases, such as independent and identically 156 distributed branches, the Rayleigh, the Nakagami and the 157 $\kappa-\mu$ fading.

4) The impact of the various channel parameters such as $\eta, 159$ $\mu, \kappa$ and that of the number of receive antennas $N$ on 160 the EVM performance is studied along with the attainable 161 performance limits.

Our paper is organized as follows. In Section II, we present 163 the background necessary for understanding this study, which 164 includes discussions on the SIMO $\eta-\mu$ and $\kappa-\mu$ shadowed 165 channel models in Section II-A and on EVM in Section II-B. 166 Subsequently, we present our analytical characterization of the 167 EVM performance for a SIMO wireless system in Section III, 168 while in Section IV we provide our simulation results. Finally, 169 we offer our conclusions in Section V.

\section{BACKGROUND INFORMATION}

\section{A. SIMO $\eta-\mu$ and $\kappa-\mu$ Shadowed Channel Models}

For the case of a SIMO wireless channel having $N$ receive 173 antennas, the channel model is as follows [4]:

$$
\hat{\mathbf{y}}=\mathbf{h} s+\mathbf{n},
$$

where $s$ is the transmitted symbol and

$$
\begin{aligned}
\hat{\mathbf{y}} & =\left[\begin{array}{llll}
\hat{y}_{1} & \hat{y}_{2} & \cdots & \hat{y}_{N}
\end{array}\right]^{T} \\
\mathbf{h} & =\left[\begin{array}{llll}
a_{1} e^{j \theta_{1}} & a_{2} e^{j \theta_{2}} & \cdots & a_{N} e^{j \theta_{N}}
\end{array}\right]^{T} \\
\mathbf{n} & =\left[\begin{array}{llll}
n_{1} & n_{2} & \cdots & n_{N}
\end{array}\right]^{T} .
\end{aligned}
$$

Here $\hat{y}_{k}$ is the symbol received by the $k^{t h}$ receive antenna after 176 being subjected to the multiplicative fading of $a_{k} e^{j \theta_{k}}$ and to 177 corruption by the additive noise of $n_{k}$. In the above discussions 178 $a_{k}, \theta_{k}$ and $n_{k}$ are random variables (RVs), whose pdf has to 179 be experimentally characterized. Typically the noise is mod- 180 elled by a zero-mean Gaussian distribution, while the phase of 181

\footnotetext{
${ }^{2}$ Note that data-aided EVM refers to the EVM obtained using data-aided receivers, i.e receivers which have exact knowledge of the transmitted bits.

${ }^{3}$ Please note that if any other detector than the MRC is used, then the EVM analysis will change significantly.
} 
the fading co-efficient is assumed to have a uniform distribution within $[0,2 \pi][4]$. However, modelling the distribution of $a_{k}$ or alternatively that of $X_{k} \propto a_{k}^{2}$ is much more challenging due to its heavy dependence on the exact nature of the wireless channel. Note that $X_{k}$ is referred to as the fading power.

Recently $\eta-\mu$ and $\kappa-\mu$ shadowed pdfs were proposed in [10] and [11], respectively. Mathematically, the $\eta-\mu$ fading power (or fading attenuation) pdf is expressed as follows for each $X_{k}$ [10], [25]:

$$
\begin{aligned}
f_{X_{k}, \eta-\mu}(x)= & \frac{2 \sqrt{\pi} \mu_{k}^{\mu_{k}+\frac{1}{2}} h_{k}^{\mu_{k}}}{\Gamma\left(\mu_{k}\right) H_{k}^{\mu_{k}-\frac{1}{2}} \bar{x}_{k}^{\mu_{k}+\frac{1}{2}}} x^{\mu_{k}-\frac{1}{2}} e^{\frac{-2 \mu_{k} h_{k} x}{\bar{x}_{k}}} . \\
& \times I_{\mu_{k}-\frac{1}{2}}\left(\frac{2 \mu_{k} H_{k} x}{\bar{x}_{k}}\right),
\end{aligned}
$$

where the modified Bessel function of the first kind of order $b$ is represented by $I_{b}($.$) and the Gamma function is denoted by$ $\Gamma$ (.) [10]. Here we have $\mu_{k}=\frac{E^{2}\left\{X_{k}\right\}}{2 \operatorname{var}\left\{X_{k}\right\}}\left[1+\left(\frac{H_{k}}{h_{k}}\right)^{2}\right]$, where $E\{$. and $\operatorname{var}\{$.$\} denote the expectation and variance, respectively and$ $\bar{x}_{k}=E\left\{X_{k}\right\}$ [25]. The parameters $H_{k}$ and $h_{k}$ may be defined in two unique ways that correspond to two distinct fading formats, where the difference arises from the physical interpretation of the parameter $\eta_{k}[10]^{4}$. In format $1,0<\eta_{k}<\infty$ is the power ratio of the in-phase and quadrature phase components of the fading signal in each multipath cluster, while $H_{k}$ and $h_{k}$ are given by:

$$
H_{k}=\frac{\eta_{k}^{-1}-\eta_{k}}{4} \text { and } h_{k}=\frac{2+\eta_{k}^{-1}+\eta_{k}}{4} .
$$

Moreover, in format 1 , the $\eta-\mu$ power distribution is symmetrical around $\eta_{k}=1$. The second format can be obtained from the first one using the relationship of $\eta_{\text {format } 2}=\frac{1-\eta_{\text {format } 1}}{1+\eta_{\text {format } 1}}[10]$.

On the other hand, the $\kappa-\mu$ shadowed power pdf is expressed as follows for each $X_{k}$ [11]:

$$
\begin{aligned}
f_{X_{k}, \kappa_{k}-\mu_{k} s h}(x)= & \frac{\mu_{k}^{\mu_{k}} m_{k}^{m_{k}}\left(1+\kappa_{k}\right)^{\mu_{k}} x^{\mu_{k}-1}}{\Gamma\left(\mu_{k}\right)\left(\bar{x}_{k}\right)^{\mu_{k}}\left(\mu_{k} \kappa_{k}+m_{k}\right)^{m_{k}}} \\
& \times e^{-\frac{\mu_{k}\left(1+\kappa_{k}\right) x}{\bar{x}_{k}}} 1 F_{1}\left(m_{k}, \mu_{k}, \frac{\mu_{k}^{2} \kappa_{k}\left(1+\kappa_{k}\right)}{\mu_{k} \kappa_{k}+m_{k}} \frac{x}{\bar{x}_{k}}\right),
\end{aligned}
$$

where $\kappa_{k}>0$ denotes the ratio of the total power of the dominant components to that of the scattered waves and $m_{k}$ is the shadowing parmeter. In (5), $\mu_{k}=\frac{E^{2}\left\{X_{k}\right\}}{\operatorname{var}\left\{X_{k}\right\}} \frac{1+2 \kappa_{k}}{\left(1+\kappa_{k}\right)^{2}}$ and $\bar{x}=$ $E\left\{X_{k}\right\}$, while ${ }_{1} F_{1}$ is the Kummer Hypergeometric function.

The elements $a_{k}$ for $1 \leq k \leq N$ have two important characteristics, which are as follows [4]:

1) Similarity: For a particular distribution model, the coefficients $a_{k}$ may or may not be identically distributed. Specifically, for the cases of the $\eta-\mu$ and $\kappa-\mu$ shadowed distributions, they may or may not all have the same $\left\{\eta_{k}, \mu_{k}\right\}$ and $\left\{\kappa_{k}, \mu_{k}, m_{k}\right\}$ parameters, respectively.

\footnotetext{
${ }^{4}$ It is important to note that the $\eta-\mu$ pdf well models the small-scale variations of the fading signal in a scenario of non-line-of-sight communication [10].
}

2) Correlation: For a particular distribution model, the coef- 218 ficients $a_{k}$ associated with $1 \leq k \leq N$ may or may not 219 be correlated with each other. The level of correlation is 220 represented by the correlation matrix as follows:

$$
\mathbf{C}_{\mathbf{m}}=\left[\begin{array}{cccccc}
\rho_{11} & \rho_{12} & \cdots & \rho_{1 j} & \cdots & \rho_{1 N} \\
\vdots & \vdots & \vdots & \rho_{i j} & \vdots & \vdots \\
\rho_{N 1} & \rho_{N 2} & \cdots & \rho_{1 j} & \cdots & \rho_{N N}
\end{array}\right],
$$

where $\rho_{i j}$ denote the correlation coefficient between $a_{i} \quad 222$ and $a_{j}$. Note that $\mathbf{C}_{\mathbf{m}}$ is an identity matrix for the case of 223 all fading magnitudes being independent.

In our study, we employ Maximal Ratio Combining 225 (MRC) [4] detection, because its performance closely matches 226 the performance of the optimal maximum-likelihood detec- 227 tion [4], while its complexity is much lower. Assuming perfect 228 channel estimation, the received symbol $y$ after MRC is as 229 follows:

$$
y=\frac{\mathbf{h}^{H} \hat{\mathbf{y}}}{\mathbf{h}^{H} \mathbf{h}} .
$$

\section{B. Error Vector Magnitude}

The error vector between the transmitted complex-valued 232 symbol $s(l)=s_{I}(l)+j \cdot s_{Q}(l)$ and the received symbol $y(l)=233$ $y_{I}(l)+j \cdot y_{Q}(l)$ is defined as $e(l)=y(l)-s(l)$. Fig. 1 shows 234 a vectorial representation of $e$ using the constellation diagram 235 of the communication system. The EVM of the communication 236 system is proportional to the root mean square value of the error 237 signal $e(l)$. In other words, if a total of $L$ symbols are trans- 238 mitted over the wireless channel, then the EVM of the SIMO 239 system described in Section II-A may be expressed as follows 240 [24]:

$$
E V M=\sqrt{\frac{\frac{1}{L} \sum_{l=1}^{L}|y(l)-s(l)|^{2}}{P_{o}}},
$$

where $P_{o}$ is the average symbol power. If $s(l) \in 242$ $\left\{S_{1}, S_{2}, \cdots S_{M}\right\}$, and if all symbols are equi-probable, 243 then $P_{o}$ may be expressed as:

$$
P_{o}=\frac{\sum_{m=1}^{M}\left|S_{m}\right|^{2}}{M} .
$$

\section{AnAlytical StUdy OF THE EVM FOR SIMO CHANNELS}

The EVM in an AWGN SISO channel has been formulated 247 as follows for the case of data-aided receivers [23]:

$$
\mathrm{EVM}=\sqrt{\frac{1}{S N R_{\text {SISO }}}} \text { when } L \rightarrow \infty,
$$

where $S N R_{S I S O}$ is the channel's signal-to-noise-ratio at the 249 single receive antenna, $L$ is the number of symbols transmit- 250 ted over the wireless channel and $M$ is the number of unique 251 wireless symbols in the modulation scheme. 


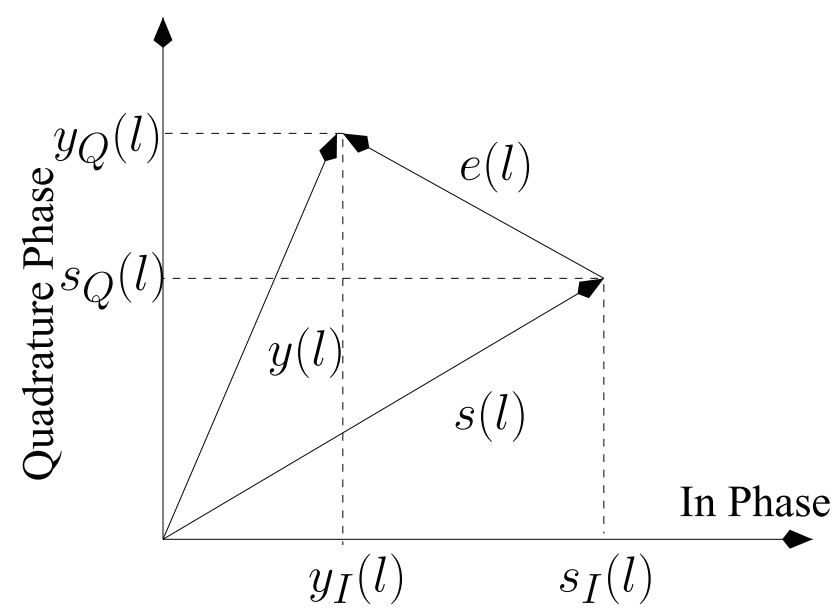

Fig. 1. Vector representation of the error between symbols $s$ and $y$.

In the SIMO scenario, if we assume that the average signal to noise ratio at each receive antenna is $\gamma_{i}=\gamma$, then the instantaneous equivalent signal-to-noise-ratio of the overall SIMO system is [4], [24]:

$$
\gamma_{\text {inst }}=\sum_{k=1}^{N} a_{k}^{2} \gamma=N \gamma \times \frac{1}{N} \sum_{k=1}^{N} a_{k}^{2}=S N R_{\text {SIMO }} \times Z,
$$

where $S N R_{S I M O}=N \gamma$ is the average equivalent signal-tonoise-ratio of the overall SIMO system, which represents the power gain of using a higher number of receivers ${ }^{5}$. On the other hand, in (11), $Z=\frac{1}{N} \sum_{k=1}^{N} a_{k}^{2}$ is the diversity gain, which converges to 1 as the number of antennas increases (assuming each of the channel gains is normalized to have unit variance) and it hence helps overcome fading [9, P. 72]. In our simulations, we compare the EVM obtained in a SIMO channel to that of the SISO channel. Our goal is to study the diversity gain obtained by employing multiple receive antennas and not the power gain. Hence, we compare the SIMO channel to an equivalent SISO AWGN channel having a signal-to-noiseratio of $S N R_{S I S O}=S N R_{S I M O}=N \gamma$ in order to ensure the same average received power in both scenearios. Note that the BER or EVM performance of the SIMO system may be better than that of a SISO AWGN channel having an $S N R_{S I S O}=\gamma$, but will always be worse than that of a SISO AWGN channel having an $S N R_{S I S O}=S N R_{S I M O}=N \gamma$.

Now, employing the instantaneous SNR in (10) we obtain the instantaneous $\operatorname{EVM}$ to be $\operatorname{EVM}(z)=\sqrt{\frac{1}{S N R_{S I M O} \times z}}$ for $L \rightarrow$ $\infty$, where $\operatorname{EVM}(z)$ is the instantaneous $\operatorname{EVM}$ for the scenario of the diversity gain $Z=z$. The average EVM is formulated by employing the definition in [24] where, the average EVM is calculated by averaging over all possible values of $z$ using the following expression:

$$
\operatorname{EVM}=\int_{0}^{\infty} \operatorname{EVM}(z) f_{Z}(z) \mathrm{d} z
$$

\footnotetext{
${ }^{5}$ We employ the notation $S N R_{S I M O}$ for distinguishing between the power gain and diversity gain obtained by employing multiple receive antennas.
}

where $f_{Z}(z)$ is the pdf of $Z$. Let us now derive the exact 282 closed-form expressions for the EVM in a SIMO channel, while 283 considering two fading scenarios, namely the $\eta-\mu$ and $\kappa-\mu 284$ shadowed fading channels.

\section{A. $\eta$ - $\mu$ Fading SIMO Channel}

286

In order to derive the EVM for $\eta$ - $\mu$ fading, we first have to 287 derive the pdf of $Z=\sum_{k=1}^{N} X_{k}$, where we have $X_{k}=\frac{1}{N} a_{k}^{2} .288$ Thus, each $X_{k}$ has the pdf given in (3) with $\bar{x}_{k}=E\left\{X_{k}\right\}=\frac{1}{N} 289$ and the distribution parameters of $\left\{\eta_{k}, \mu_{k}\right\}$. The moment gen- 290 erating function (MGF) for $X_{k}$ has been derived in [26]. In 291 [27], it has been shown that the MGF of $X_{k}$ can be repre- 292 sented as the product of the MGFs of two gamma distributed 293 RVs (RVs), where both these gamma RVs have the same shape 294 parameter $\alpha_{2 k-1}=\alpha_{2 k}=\mu_{k}$, but different scale parameters 295 of $\theta_{2 k-1}=\frac{\bar{x}_{k}}{2 \mu_{k}\left(h_{k}+H_{k}\right)}$ and $\theta_{2 k}=\frac{\bar{x}_{k}}{2 \mu_{k}\left(h_{k}-H_{k}\right)}$. Using this rela- 296 tionship, as well as the studies in [27] and [28], we can state 297 that $X_{k}=P_{k}+Q_{k}$, such that $P_{k} \sim \mathcal{G}\left(\alpha_{2 k-1}, \theta_{2 k-1}\right)$, and $Q_{k} \sim 298$ $\mathcal{G}\left(\alpha_{2 k}, \theta_{2 k}\right)$. Note that $\mathcal{G}\left(\alpha_{2 k}, \theta_{2 k}\right)$ denote the gamma distribu- 299 tion with shape parameter $\alpha_{2 k}$ and scale parameter $\theta_{2 k}$. Thus, 300 the sum of $N \eta$ - $\mu \mathrm{RVs}$ may be alternatively expressed as the 301 sum of $L=2 N$ Gamma RVs, where the pdf of the sum of $L 302$ Gamma RVs has been derived in [29]. Now, as stated earlier, 303 the pdf of $Z=\sum_{k=1}^{N} X_{k}$ has been derived to be the following 304 using the pdf of the sum of $2 N$ Gamma RVs [29]:

$$
\begin{aligned}
& f_{Z, \eta-\mu}(z)=\frac{\sum^{\sum_{i=1}^{2 N} \alpha_{i}-1}}{\prod_{i=1}^{2 N}\left(\theta_{i}\right)^{\alpha_{i}} \Gamma\left(\sum_{i=1}^{2 N} \alpha_{i}\right)} \\
& \Phi_{2}^{(2 N)}\left(\alpha_{1}, \cdots, \alpha_{2 N} ; \sum_{i=1}^{2 N} \alpha_{i} ; \frac{-z}{\theta_{1}}, \cdots, \frac{-z}{\theta_{2 N}}\right),
\end{aligned}
$$

where $\Phi_{2}^{(N)}$ (.) is the confluent Lauricella function [30]. We 306 can now substitute this $f_{Z, \eta-\mu}(z)$ into (12) for formulating the 307 average EVM.

1) EVM of $\eta-\mu$ SIMO Channel With Independent and 309 Nonidentically Distributed Branches:

Lemma 1: The EVM expression of the $\eta-\mu$ fading SIMO 311 channel having independent and non-identically distributed 312 (i.n.i.d) branches is given by

$$
\begin{gathered}
E V M_{\eta-\mu, i . n . i . d}=\frac{\sqrt{N \mu_{1}\left(1+\eta_{1}^{-1}\right)}}{\sqrt{S N R_{S I M O}}} \frac{\Gamma\left(2 \sum_{i=1}^{N} \mu_{i}-0.5\right)}{\Gamma\left(2 \sum_{i=1}^{N} \mu_{i}\right)} \\
\times F_{D}^{(2 N-1)}\left[0.5, \mu_{1}, \mu_{2}, \mu_{2}, \cdots, \mu_{N}, \mu_{N} ; 2 \sum_{i=1}^{N} \mu_{i}\right. \\
1-\frac{1}{\eta_{1}}, 1-\frac{\mu_{1}\left(1+\eta_{1}^{-1}\right)}{\mu_{2}\left(1+\eta_{2}^{-1}\right)}, 1-\frac{\mu_{1}\left(1+\eta_{1}^{-1}\right)}{\mu_{2}\left(1+\eta_{2}\right)} \cdots \\
\left.1-\frac{\mu_{1}\left(1+\eta_{1}^{-1}\right)}{\mu_{N}\left(1+\eta_{N}^{-1}\right)}, 1-\frac{\mu_{1}\left(1+\eta_{1}^{-1}\right)}{\mu_{N}\left(1+\eta_{N}\right)}\right]
\end{gathered}
$$

for $2 \sum_{i=1}^{N} \mu_{i}>0.5$ 
Proof: See Appendix I for the proof.

The expression of the EVM for the i.n.i.d case is given in terms of Lauricella's function of the fourth kind $F_{D}^{(N)}[$. [30]. The function $F_{D}^{(N)}\left[a, b_{1}, \cdots, b_{N} ; c ; x_{1}, \cdots, x_{N}\right]$ can be evaluated using the following single integral expression:

$$
\frac{\Gamma(c)}{\Gamma(a) \Gamma(c-a)} \int_{0}^{1} t^{a-1}(1-t)^{c-a-1} \prod_{i=1}^{N}\left(1-x_{i} t\right)^{-b_{i}} \mathrm{~d} t,
$$$$
\text { where } \operatorname{Real}(c)>\operatorname{Real}(a)>0,
$$

where Real(.) returns the real part of the argument. Note that the condition $\operatorname{Real}(c)>\operatorname{Real}(a)>0$ is satisfied by Lauricella's function of the fourth kind $F_{D}^{(N)}$ [.], which appeared in (14).

Special Case 1: Now, we simplify the expression in (14) for the case of independent and identically distributed (i.i.d) fading SIMO channels. Substituting both $\eta_{i}=\eta$ and $\mu_{i}=\mu \forall i$ into (14) and then using the following identity:

$F_{D}^{(N)}\left[a, b_{1}, \cdots b_{n} ; c, x, \cdots, x\right]=2 F_{1}\left[a, b_{1}+\cdots+b_{N} ; c ; x\right]$,

where ${ }_{2} F_{1}[$.$] is the Gauss hypergeometric function [30], we$ obtain,

$$
\begin{aligned}
& \mathrm{EVM}_{\eta-\mu, i . i . d}=\frac{\sqrt{N \mu\left(1+\eta^{-1}\right)}}{\sqrt{S N R_{S I M O}}} \frac{\Gamma(2 N \mu-0.5)}{\Gamma(2 N \mu)} \\
& { }_{2} F_{1}\left[0.5, N \mu ; 2 N \mu ; 1-\frac{1}{\eta}\right] \text { for } 2 N \mu>0.5 .
\end{aligned}
$$

In the following, we show that the expression shown in (17) converges to the EVM expression of AWGN channel. Note that when fading parameters $\eta=1$ and $\mu$ tends to infinity, the $\eta-\mu$ channel should converge to an AWGN channel. By substituting $\eta=1$ and $\mu \rightarrow \infty$ in (17), it can be simplified to

$$
\begin{aligned}
\operatorname{EVM}_{A W G N} & =\lim _{\mu \rightarrow \infty} \frac{\sqrt{2 N \mu}}{\sqrt{S N R_{S I M O}}} \frac{\Gamma(2 N \mu-0.5)}{\Gamma(2 N \mu)} \\
& =\frac{1}{\sqrt{S N R_{S I M O}}} .
\end{aligned}
$$

This simplification follows from the fact that ${ }_{2} F_{1}[0.5, N \mu ; 2 N \mu ; 0]=1$. We now provide the upper bound of the EVM expression given in (17) so that the impact of fading parameter $\eta$ can be shown. Using the transformation ${ }_{2} F_{1}[a, b ; c ; z]=(1-z)_{2}^{c-a-b} F_{1}[c-a, c-b ; c ; z]$, we obtain:

$$
\begin{aligned}
& \mathrm{EVM}_{\eta-\mu, i . i . d}=\frac{\sqrt{N \mu\left(1+\eta^{-1}\right)}}{\sqrt{S N R_{S I M O}}} \frac{\Gamma(2 N \mu-0.5)}{\Gamma(2 N \mu)} \\
& { }_{2} F_{1}\left[2 N \mu-0.5, N \mu ; 2 N \mu ; 1-\frac{1}{\eta}\right]\left(\frac{1}{\eta}\right)^{\mu-0.5}
\end{aligned}
$$

340 and using the bound ${ }_{2} F_{1}\left[2 N \mu-0.5, N \mu ; 2 N \mu ; 1-\frac{1}{\eta}\right]<2$ $341 F_{1}\left[2 N \mu, N \mu ; 2 N \mu ; 1-\frac{1}{\eta}\right]\left(\frac{1}{\eta}\right)^{-\mu}$, the expression given in
(19) can be upper bounded as:

$$
\begin{aligned}
\mathrm{EVM}_{\eta-\mu, i . i . d} & <\frac{\sqrt{N \mu\left(1+\eta^{-1}\right)}}{\sqrt{S N R_{S I M O}}} \frac{\Gamma(2 N \mu-0.5)}{\Gamma(2 N \mu)}\left(\frac{1}{\eta}\right)^{-0.5} \\
& =\frac{\sqrt{N \mu(1+\eta)}}{\sqrt{S N R_{S I M O}}} \frac{\Gamma(2 N \mu-0.5)}{\Gamma(2 N \mu)}
\end{aligned}
$$

Hence, it is apparent from (20) that as $\eta$ increases, the EVM 343 increases. Recall that $\eta$ is the scattered-wave power ratio 344 between the in-phase and quadrature components of each clus- 345 ter of multipath and hence the EVM will be minimum when the 346 power of the in-phase and the quadrature components is equal. 347

Special Case 2: The Nakagami-m fading is a special case 348 of the $\eta-\mu$ fading associated with $\eta=1$ and $2 \mu=m^{\prime}$. Note 349 that $m^{\prime}$ is the shape parameter of the Nakagami- $m$ fading. 350 Substituting $\eta=1$ and $2 \mu=m$ in (14) and (17), we obtain 351 the following expressions for the i.n.i.d and i.i.d scenarios, 352 respectively:

$\mathrm{EVM}_{n, i . n . i . d}=\frac{\sqrt{N m_{1}^{\prime}}}{\sqrt{S N R_{S I M O}}} \frac{\Gamma\left(\sum_{i=1}^{N} m_{i}^{\prime}-0.5\right)}{\Gamma\left(\sum_{i=1}^{N} m_{i}^{\prime}\right)} \times$

$F_{D}^{(N-1)}\left[0.5, m_{2}^{\prime}, \cdots, m_{N}^{\prime} ; \sum_{i=1}^{N} m_{i}^{\prime} ; 1-\frac{m_{1}^{\prime}}{m_{2}^{\prime}}, \cdots, 1-\frac{m_{1}^{\prime}}{m_{N}^{\prime}}\right]$

for $\sum_{i=1}^{N} m_{i}^{\prime}>0.5$ and

$\mathrm{EVM}_{n, \text { i.i.d }}=\frac{\sqrt{N m^{\prime}}}{\sqrt{S N R_{\text {SIMO }}}} \frac{\Gamma\left(N m^{\prime}-0.5\right)}{\Gamma\left(N m^{\prime}\right)}$ for $N m^{\prime}>0.5$.

Using the following identity from [31]:

$$
\begin{aligned}
& \frac{\Gamma(n+a)}{\Gamma(n+b)}=n^{a-b}\left(1+\frac{(a-b)(a+b-1)}{2 n}+O\left(|n|^{-2}\right)\right) \\
& \quad \text { for large } n,
\end{aligned}
$$

the EVM of the i.i.d Nakagami-m scenario can be further 355 simplified to

$$
\begin{gathered}
\mathrm{EVM}_{n, \text { i.i.d }}=\frac{1}{\sqrt{S N R_{S I M O}}}\left(1+\frac{0.75}{2 N m^{\prime}}+O\left(\left|N m^{\prime}\right|^{-2}\right)\right) \\
\text { for large } N m^{\prime} .
\end{gathered}
$$

Note that the first term in (24) represents the EVM of an 357 AWGN channel, while the remaining terms in (24) represent 358 the contribution of the fading. We know that as the parameter $m 359$ decreases, the impact of fading becomes more severe, which is 360 confirmed by (24). A second point that may be noted from (24) 361 is that the EVM approaches that of an AWGN channel, when 362 the number of receive antennas tends to infinity and/or when 363 the fading parameter tends to infinity. 364

2) EVM of $\eta-\mu$ SIMO Channel With Correlated and 365 Identically Distributed Branches: $\quad 366$

Lemma 2: The EVM expression of a correlated $\eta-\mu$ SIMO 367 channel associated with an MRC-based receiver is given by: 368 


$$
\begin{aligned}
& \mathrm{EVM}_{\eta-\mu, \text { corr }}=\frac{1}{\sqrt{\hat{\theta}_{1} S N R_{S I M O}}} \frac{\Gamma\left(2 N \mu_{c}-0.5\right)}{\Gamma\left(2 N \mu_{c}\right)} \\
& \quad \times F_{D}^{(2 N-1)}\left(0.5, \mu_{c}, \cdots, \mu_{c} ; 2 N \mu_{c} ; 1-\frac{\hat{\theta}_{2}}{\hat{\theta}_{1}}, \cdots 1-\frac{\hat{\theta}_{2 N}}{\hat{\theta}_{1}}\right) \\
& \quad \text { for } 2 N \mu_{c}>0.5 .
\end{aligned}
$$

Proof: See Appendix II for the proof.

\section{B. $\kappa-\mu$ Shadowed Fading SIMO Channel}

In order to derive the EVM of a $\kappa-\mu$ shadowed faded channel, we first have to derive the pdf of $Z=\sum_{k=1}^{N} X_{k}$, where $X_{k}=$ $\frac{1}{N} a_{k}^{2}$. Thus, each $X_{k}$ has the pdf given in (5) with $\bar{x}_{k}=\frac{1}{N}$ and distribution parameters of $\left\{\kappa_{k}, \mu_{k}, m_{k}\right\}$. The pdf of $Z$ has been shown in [11] to be as follows:

$$
\begin{aligned}
& f_{Z, \kappa-\mu s h}(z)=\prod_{i=1}^{N} \frac{\left.\mu_{i}^{\mu_{i}} m_{i}^{m_{i}}\left(1+\kappa_{i}\right)^{\mu_{i}} z^{i=1}{ }^{N} \mu_{i=1}^{N} \mu_{i}\right)\left(\mu_{i} \kappa_{i}+m_{i}\right)^{m_{i}} \bar{x}_{i}^{\mu_{i}}}{\Phi_{2}^{(N)}\left(\mu_{1}-m_{1}, \cdots \mu_{N}-m_{N}, m_{1} \cdots m_{n} ; \sum_{i=1}^{N} \mu_{i} ;\right.} \\
& -\frac{\mu_{1}\left(1+\kappa_{1}\right) z}{\bar{x}_{1}}, \cdots,-\frac{\mu_{N}\left(1+\kappa_{N}\right) z}{\bar{x}_{N}},-\frac{\mu_{1} m_{1}\left(1+\kappa_{1}\right) z}{\left(\mu_{1} \kappa_{1}+m_{1}\right) \bar{x}_{1}} \\
& \left.\cdots-\frac{\mu_{N} m_{N}\left(1+\kappa_{N}\right) z}{\left(\mu_{N} \kappa_{N}+m_{N}\right) \bar{x}_{N}}\right) .
\end{aligned}
$$

We can now substitute $f_{Z, \kappa-\mu s h}(z)$ in (12) to obtain the average EVM.

1) EVM of $\kappa-\mu$ Shadowed Fading SIMO Channel With i.n.i.d Branches:

Lemma 3: The EVM expression of $\kappa-\mu$ shadowed fading SIMO channel having i.n.i.d branches is given by

$$
\begin{aligned}
& E V M_{\kappa-\mu s h, i . n . i . d}=\frac{\sqrt{N \mu_{1}\left(1+\kappa_{1}\right)}}{\sqrt{S N R_{S I M O}}} \frac{\Gamma\left(\sum_{i=1}^{N} \mu_{i}-0.5\right)}{\Gamma\left(\sum_{i=1}^{N} \mu_{i}\right)} \\
& F_{D}^{(2 N-1)}\left(0.5, \mu_{2}-m_{2}, \cdots, \mu_{2}-m_{2}, m_{1}, \cdots m_{N} ;\right. \\
& \sum_{i=1}^{N} \mu_{i} ; 1-\frac{\mu_{1}\left(1+\kappa_{1}\right)}{\mu_{2}\left(1+\kappa_{2}\right)}, \cdots, 1-\frac{\mu_{1}\left(1+\kappa_{1}\right)}{\mu_{N}\left(1+\kappa_{N}\right)}, \\
& \left.1-\frac{\left(\mu_{1} \kappa_{1}+m_{1}\right)}{m_{1}} \cdots 1-\frac{\left(\mu_{N} \kappa_{N}+m_{N}\right) \mu_{1}\left(1+\kappa_{1}\right)}{m_{N} \mu_{N}\left(1+\kappa_{N}\right)}\right), \\
& \text { for } \sum_{i=1}^{N} \mu_{i}>0.5 .
\end{aligned}
$$

Proof: See Appendix III for the proof.

Special Case 1: Now, we simplify the expression in (27) for the i.i.d scenario, where we set $\mu_{i}=\mu$ and $\kappa_{i}=\kappa \forall i$ to obtain:

$$
\begin{aligned}
& \mathrm{EVM}_{\kappa-\mu s h, i . i . d}=\frac{\sqrt{N \mu(1+\kappa)}}{\sqrt{S N R_{S I M O}}} \frac{\Gamma(N \mu-0.5)}{\Gamma(N \mu)} \\
& { }_{2} F_{1}\left[0.5, N m ;, N \mu ;-\frac{\mu \kappa}{m}\right] \text { for } N \mu>0.5 .
\end{aligned}
$$

In the following, we will show that the above expression con- 386 verges to the EVM expression of AWGN channel. Note that 387 when fading parameters $\kappa=0$ and $\mu$ tends to infinity, the $\kappa$ - 388 $\mu$ channel should converge to an AWGN channel. By putting 389 $\kappa=0$ and $\mu \rightarrow \infty$ the above expression can be simplified to 390

$$
\begin{aligned}
\mathrm{EVM}_{A W G N} & =\lim _{\mu \rightarrow \infty} \frac{\sqrt{N \mu}}{\sqrt{S N R_{S I M O}}} \frac{\Gamma(N \mu-0.5)}{\Gamma(N \mu)} \\
& =\frac{1}{\sqrt{S N R_{S I M O}}} .
\end{aligned}
$$

The above simplification follows from the fact that 391 ${ }_{2} F_{1}[0.5, N \mu ; 2 N \mu ; 0]=1$. Note that the EVM expres- 392 sion for an $\kappa-\mu$ shadowed channel converges to the expression 393 of AWGN channel when fading parameter $\kappa=0$ and $\mu$ tends 394 to infinity, as expected.

Special Case 2: We now derive the closed-form expression 396 of EVM for the $\kappa-\mu$ fading SIMO channel having i.i.d branches. 397 Note that the $\kappa-\mu$ fading is a special case of the $\kappa-\mu$ shadowed 398 fading with $m \rightarrow \infty$. Using the following identity [32]:

$$
\lim _{b \rightarrow \infty}{ }_{2} F_{1}\left[a, b, c, \frac{z}{b}\right]={ }_{1} F_{1}[a, c, z],
$$

the ${ }_{2} F_{1}[$.$] given in (28) can be simplified for m \rightarrow \infty$ as 400 follows:

$$
{ }_{2} F_{1}\left[0.5, N m ;, N \mu ;,-\frac{\mu \kappa}{m}\right]=1 F_{1}[0.5 ; N \mu ;-N \mu \kappa],
$$

where ${ }_{1} F_{1}[$.$] is the Kummer hypergeometric function [30]. 402$ Therefore, the EVM expression of the $\kappa-\mu$ fading SIMO chan- 403 nel having i.i.d branches is given by

$$
\begin{aligned}
& \mathrm{EVM}_{\kappa-\mu, i . i . d}=\frac{\sqrt{N \mu(1+\kappa)}}{\sqrt{S N R_{S I M O}}} \frac{\Gamma(N \mu-0.5)}{\Gamma(N \mu)} \\
& { }_{1} F_{1}(0.5, N \mu,-N \mu \kappa) \text { for } N \mu>0.5 .
\end{aligned}
$$

The EVM expression of the $\kappa-\mu$ fading SISO channel is 405 given by

$$
\begin{gathered}
\mathrm{EVM}_{\kappa-\mu}=\frac{\sqrt{\mu(1+\kappa)}}{\sqrt{S N R}} \frac{\Gamma(\mu-0.5)}{\Gamma(\mu)} 1 F_{1}(0.5, \mu,-\mu \kappa) \\
\text { for } \mu>0.5 .
\end{gathered}
$$

Additional validation of Equation (33): In the following, we 407 derive the EVM expression of the $\kappa-\mu$ fading SISO channel 408 using the negative moment given in [10] in order to fur- 409 ther validate our derivations ${ }^{6}$. The EVM for $\kappa-\mu$ channel is 410

\footnotetext{
${ }^{6}$ Note that the negative moment of sum of generalized fading distribution is not available and hence we cannot derive the EVM expression for SIMO channel.
} 
given by

$$
\operatorname{EVM}_{\kappa-\mu}=\int_{a=0}^{\infty} \sqrt{\frac{1}{a^{2} \mathrm{SNR}}} f_{\kappa-\mu}(a) \mathrm{d} a
$$

where $f_{\kappa-\mu}(a)$ is the $\kappa-\mu$ envelope probability density function. It is apparent from the above expression that the $\mathrm{EVM}_{\kappa-\mu}$ is the negative moment of the $\kappa-\mu$ fading distribution. Using the moment expression given in [10], the $\mathrm{EVM}_{\kappa-\mu}$ can be obtained as

$$
\begin{aligned}
& \operatorname{EVM}_{\kappa-\mu}=\frac{\sqrt{\mu(1+\kappa)}}{\sqrt{S N R}} \frac{\Gamma(\mu-0.5) \exp (-\kappa \mu)}{\Gamma(\mu)} \\
& { }_{1} F_{1}(\mu-0.5, \mu, \mu \kappa) \text { for } \mu>0.5 .
\end{aligned}
$$

Then, using the transformation $e_{1}^{-z} F_{1}(b-a, b, z)$ $=1 F_{1}(a, b,-z)$, we can simplify the above expression to

$$
\mathrm{EVM}_{\kappa-\mu}=\frac{\sqrt{\mu(1+\kappa)}}{\sqrt{S N R}} \frac{\Gamma(\mu-0.5)}{\Gamma(\mu)} 1 F_{1}(0.5, \mu,-\mu \kappa) .
$$

Therefore, we have shown that the expressions given in (35) and (33) are same. Note that the functional form of the pdf of the sum of correlated $\kappa-\mu$ shadowed random variables is similar to that of the sum of correlated $\eta-\mu$ random variables. Hence, the EVM expression for a correlated $\kappa-\mu$ shadowed SIMO channel can be derived in a similar manner to that of the $\eta-\mu$ SIMO channel. Furthermore, $\kappa-\mu$ fading is a special case of $\kappa-\mu$ shadowed fading and hence the EVM can be obtained numerically by employing a very high value of $m$ in the EVM expression for a $\kappa-\mu$ shadowed fading channel.

\section{Simulation Results}

In order to validate the EVM expressions derived for $\eta$ $\mu$ and $\kappa-\mu$ shadowed fading channels associated with the arbitary parameters, we simulated a BPSK modulation-based system communicating over these channels. We implemented a simulation-based solution of (12) using 1 transmit and $N$ receive antennas. The simulations employed the Monte Carlo approach, which relies on transmitting a large number of bits over the wireless channel and computing the average EVM. The simulations were carried out in Matlab.

Fig. 2 shows the EVM variation with respect to $S N R_{S I M O}$ for the case of SIMO channels having independent and nonidentically distributed branches, where it can be seen that the simulation results closely match the theoretical values.

Fig. 3 depicts the variation of EVM with respect to $S N R_{S I M O}$ for $\eta-\mu$ fading. Here, we have considered $N=3$ and $\eta \geq 1$, since $\eta$ is symmetrical about 1 . Firstly, it may be seen that the analytical results match with the simulation results for the entire range of $S N R_{S I M O}$. Secondly, it may be observed that as $\eta$ increases, the EVM also increases for a fixed value of $\mu$. Recall that $\eta$ is the power ratio of the in-phase and quadrature-phase components of the fading signal in each multipath cluster. Hence, as the power ratio of the in-phase and

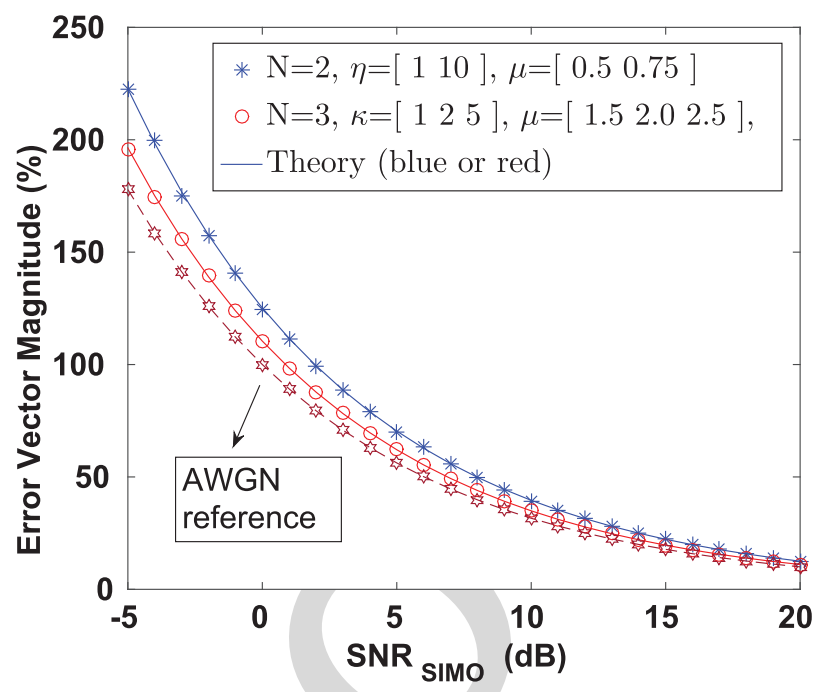

Fig. 2. The EVM for $\eta-\mu$ and $\kappa-\mu$ shadowed i.n.i.d SIMO channels.

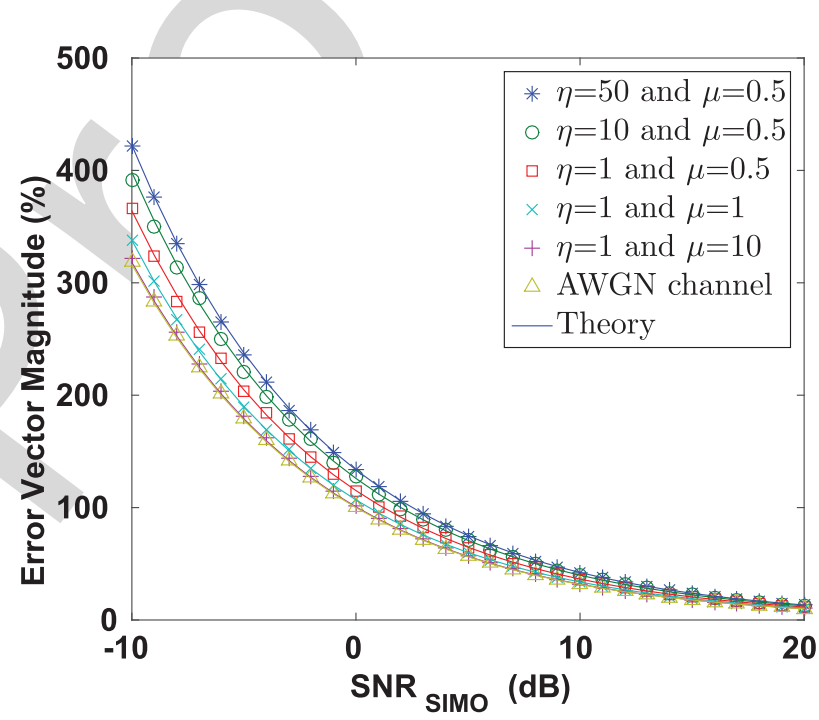

Fig. 3. The EVM for different values of $\eta$ and $\mu$, when $N=3$ and the channels are i.i.d.

quadrature-phase components increases, the EVM increases. In 453 other words, the EVM would be minimum, when the power 454 of the in-phase and quadrature-phase components of the fading 455 signal in each multipath cluster is equal. Thirdly, as the shape 456 parameter $\mu$ increases, the EVM decreases and it approaches 457 the EVM of an AWGN channel.

Fig. 4 shows the variation of EVM with respect to $S N R_{S I M O} \quad 459$ for different values of $N$. Firstly, observe that the simula- 460 tion results closely match the analytical results. Secondly, as 461 the number of antennas increases, the EVM decreases and it 462 approaches the EVM of an AWGN channel. Interestingly, it 463 may be seen that the EVM decreases significantly as the number 464 of antennas increases from 1 to 2 . However, the EVM reduction 465 becomes less significant, as the number of antennas increases 466 from $N=2$ to 3 and so on.

Fig. 5 shows the variation of EVM as a function of 468 $S N R_{S I M O}$ for different values of correlation coefficients. The 469 correlation between the SIMO branches is defined by the corre- 470 lation matrix in (6) in conjunction with $\rho_{p q}=\rho^{|p-q|}$, where 471 


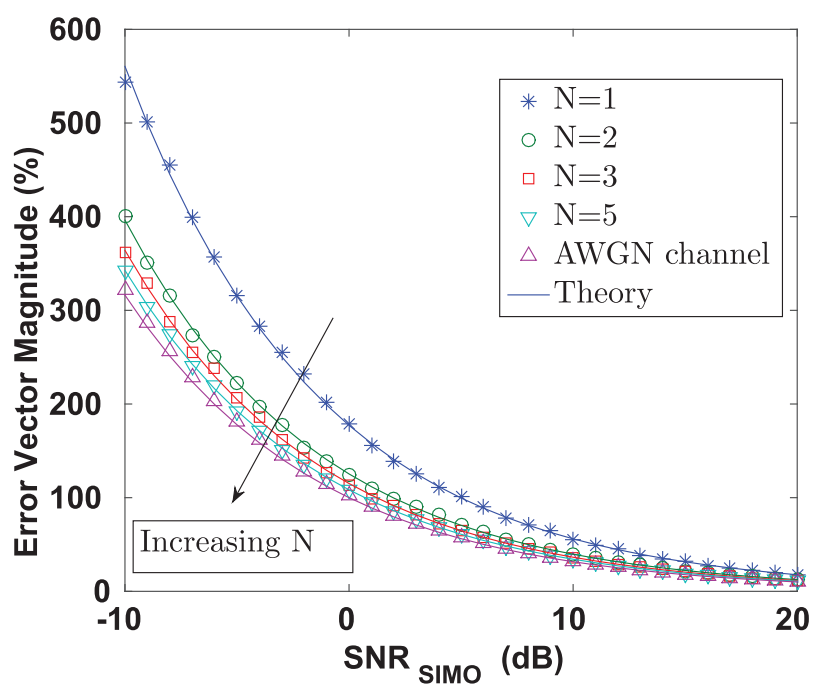

Fig. 4. The EVM for different values of $\mathrm{N}$, when $\eta=1$ and $\mu=0.5$ and the channels are i.i.d.



Fig. 5. The EVM for different values of correlation, when $N=3$, while $\eta=1$ and $\mu=0.5$ for all the channels. we have $p, q=1, \cdots, N$. Firstly, it may be seen that the simulation results closely match the analytical results for all values of the correlation coefficients. Secondly, it is observed that the EVM increases, as the correlation among the branches increases and it approaches the EVM of a SISO channel. Furthermore, the rate at which the EVM increases is higher, when the correlation coefficients are high.

Fig. 6 shows the variation of EVM versus the $N \times m$ product for the special case of Nakagami channels. It may be seen that the EVM decreases, as either $N$ or $m$ increases. Interestingly, the rate at which the EVM decreases is higher, when the number of antennas and the Nakagami-m fading parameter are small. This phenomenon may also be observed from (24), where the EVM of the Nakagami-m fading is shown to be a function of $1 /(N \times m)$.

Fig. 7 shows the EVM variation versus $S N R_{S I M O}$ for $\kappa-\mu$ fading. Again, the simulation results closely match the analytical results. It may be seen that as $\kappa$ increases, the EVM

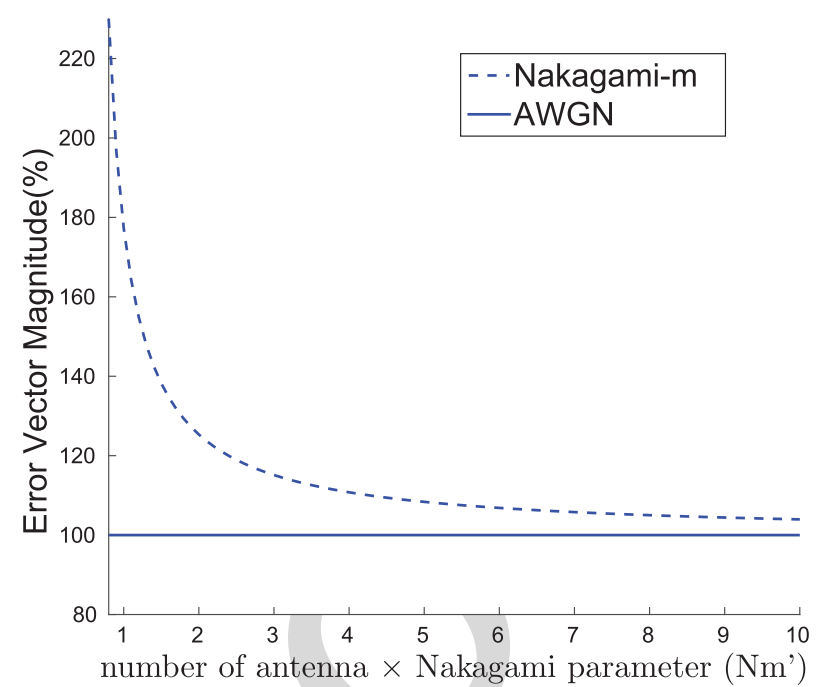

Fig. 6. Variation in EVM with respect to $N \times m^{\prime}$ for a Nakagami SIMO channel which are i.i.d.

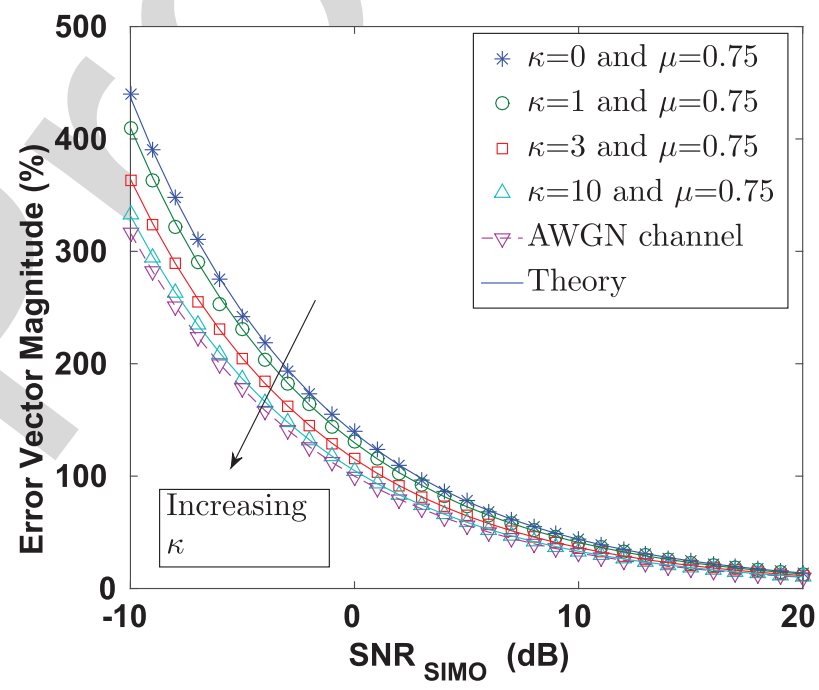

Fig. 7. The EVM for different combinations of $\kappa$ and $\mu$, when $N=2$ and the channels are i.i.d.

decreases and it approaches the EVM of an AWGN channel. 490 Recall that $\kappa$ represents the ratio of the total power of the dom- 491 inant components to that of the scattered waves. Hence, as the 492 ratio of the total power of the dominant components to that of 493 the scattered waves increases, the EVM decreases, as expected. 494

\section{Conclusions}

We have derived exact closed-form expressions for the data- 496 aided EVM in $\eta-\mu$ and $\kappa-\mu$ shadow faded SIMO channels 497 having independent and non-identically distributed branches. 498 The EVM expression is also derived for the scenario of cor- 499 related SIMO branches. Furthermore, the expressions derived 500 may be readily simplified for various special cases, such as 501 independent and identically distributed fading, the Rayleigh, 502 the Nakagami-m and finally the $\kappa-\mu$ fading. Subsequently, we 503 performed a simulation based study of this system in order to 504 validate the analytical results. Finally, a parametric study of the 505 
EVM performance of the wireless communication system considered showed that as the Nakagami fading parameter $m$ and/or the number of antennas $N$ increases, the EVM decreases and the rate at which the EVM decreases is higher, when the fading parameter and/or the number of antennas is small.

\section{ACKNOWLEDGMENT}

We are grateful to Dr. R. A. Shafik for his valuable inputs.

\section{APPENDIX A}

The EVM for a AWGN SISO channel is given by (10) [23], [24]. Thus, the instantaneous EVM, namely $\operatorname{EVM}(z)$, is computed using (10) but with the replacement of $S N R_{S I S O}$ with the instantaneous signal-to-noise ratio, where $z S N R_{S I M O}$ is the instantaneous signal-to-noise ratio as per (11). Thus, the EVM of a $\eta-\mu$ fading channel is defined as follows [24]:

$$
\operatorname{EVM}_{\eta-\mu, i . n . i . d}=\int_{0}^{\infty} \operatorname{EVM}(z) f_{Z, \eta-\mu}(z) \mathrm{d} z
$$

which simply weights the AWGN channel's EVM by the specific probability of occurance of each particular instantaneous SNR given by its distribution and then averages it by integrating it across the entire instantaneous SNR range. Now substituting (13) in (36), we get:

$$
\begin{aligned}
& \operatorname{EVM}_{\eta-\mu, i . n . i . d}=\int_{0}^{\infty} \sqrt{\frac{1}{z S N R_{S I M O}}} \frac{\sum^{i=1} \alpha_{i}-1}{\prod_{i=1}^{2 N}\left(\theta_{i}\right)^{\alpha_{i}} \Gamma\left(\sum_{i=1}^{2 N} \alpha_{i}\right)} . \\
& \Phi_{2}^{(2 N)}\left(\alpha_{1}, \cdots, \alpha_{2 N} ; \sum_{i=1}^{2 N} \alpha_{i} ; \frac{-z}{\theta_{1}}, \cdots, \frac{-z}{\theta_{2 N}}\right) \mathrm{d} z \\
& =\int_{0}^{\infty} \sqrt{\frac{1}{S N R_{S I M O}}} \frac{\sum^{i=1} \alpha_{i}-1.5}{\prod_{i=1}^{2 N}\left(\theta_{i}\right)^{\alpha_{i}} \Gamma\left(\sum_{i=1}^{2 N} \alpha_{i}\right)} \\
& \Phi_{2}^{(2 N)}\left(\alpha_{1}, \cdots, \alpha_{2 N} ; \sum_{i=1}^{2 N} \alpha_{i} ; \frac{-z}{\theta_{1}}, \cdots, \frac{-z}{\theta_{2 N}}\right) \mathrm{d} z .
\end{aligned}
$$

Using the transformation [30, P. 177]:

$$
\begin{aligned}
& e^{-x_{i}} \Phi_{2}^{(n)}\left(b_{1}, \cdots, b_{n} ; c ; x_{1}, \cdots, x_{n}\right) \\
& \quad=\Phi_{2}^{(n)}\left(b_{1}, \cdot, b_{i-1}, c-b_{1}-\cdot-b_{n}, b_{i+1}, \cdot, b_{n} ; c ;\right. \\
& \left.\quad x_{1}-x_{i}, \cdots x_{i-1}-x_{i},-x_{i}, x_{i+1}-x_{i}, \cdot, x_{n}-x_{i}\right),
\end{aligned}
$$

the $\mathrm{EVM}_{\eta-\mu, i . n . i . d}$ can be rewritten as:

$$
\begin{aligned}
& \operatorname{EVM}_{\eta-\mu, i . n . i . d}=K_{1} \int_{0}^{\infty} z^{i=1} \alpha_{i}^{2 N} \alpha^{-\frac{z}{\theta_{1}}} \\
& \quad \times \Phi_{2}^{(2 N)}\left(0, \alpha_{2}, \cdots, \alpha_{2 N} ; \sum_{i=1}^{2 N} \alpha_{i} ; \frac{z}{\theta_{1}},\left(\frac{1}{\theta_{1}}-\frac{1}{\theta_{2}}\right) z\right. \\
& \left.\cdots\left(\frac{1}{\theta_{1}}-\frac{1}{\theta_{N}}\right) z\right) \mathrm{d} z
\end{aligned}
$$

where we have $K_{1}=\sqrt{\frac{1}{S N R_{S I M O}}} \frac{1}{\prod_{i=1}^{2 N}\left(\theta_{i}\right)^{\alpha_{i}} \Gamma\left(\sum_{i=1}^{2 N} \alpha_{i}\right)}$. Note that if 527 one of the numerator parameters of the series expansion of 528 $\Phi_{2}^{(N)}$ goes to zero, then $\Phi_{2}^{(N)}$ becomes $\Phi_{2}^{(N-1)}$ and hence the 529 above $\Phi_{2}^{(2 N)}$ will become $\Phi_{2}^{(2 N-1)}$ with appropriate parameters. 530 Using the transformation $\frac{z}{\theta_{1}}=t$ we obtain:

$$
\begin{aligned}
& \operatorname{EVM}_{\eta-\mu, i . n . i . d}=K_{1} \theta_{1}^{\sum_{i=1}^{2 N} \mu_{i}-0.5} \int_{0}^{\infty} \sum^{2 N} \alpha_{i=1}^{2 N} e^{-t} \\
& \times \Phi_{2}^{(2 N-1)}\left(\alpha_{2}, \cdots, \alpha_{2 N} ; \sum_{i=1}^{2 N} \alpha_{i} ;\left(1-\frac{\theta_{1}}{\theta_{2}}\right) t,\right. \\
& \left.\quad \ldots\left(1-\frac{\theta_{1}}{\theta_{N}}\right) t\right) \mathrm{d} t .
\end{aligned}
$$

Using the following identity [30, P. 51]:

$$
\begin{aligned}
& F_{D}^{(n)}\left[a, b_{1}, \cdots, b_{n}, c, x_{1}, \cdots, x_{n}\right] \\
& =\frac{1}{\Gamma(a)} \int_{t=0}^{\infty} e^{-t} t^{a-1} \Phi_{2}^{(n)}\left[b_{1}, \cdots, b_{n}, c, x_{1} t, \cdots, x_{n} t\right] \mathrm{d} t
\end{aligned}
$$

where $\operatorname{Real}(a)>0$, one obtains:

$$
\begin{aligned}
& \operatorname{EVM}_{\eta-\mu, i . n . i . d}=K_{1} \theta_{1}^{\sum_{i=1}^{2 N} \alpha_{i}-0.5} \Gamma\left(\sum_{i=1}^{2 N} \alpha_{i}-0.5\right) \\
& \times F_{D}^{(2 N-1)}\left(\sum_{i=1}^{2 N} \alpha_{i}-0.5, \alpha_{2}, \cdots, \alpha_{2 N} ; \sum_{i=1}^{2 N} \alpha_{i}\right. \\
& \left.1-\frac{\theta_{1}}{\theta_{2}}, \cdots, 1-\frac{\theta_{1}}{\theta_{N}}\right)
\end{aligned}
$$

for $\sum_{i=1}^{2 N} \alpha_{i}>0.5$. Here $F_{D}^{(N)}\left[a, b_{1}, \cdots, b_{N} ; c ; x_{1}, \cdots, x_{N}\right]$ is 534 the Lauricella's function of the fourth kind. Again, using the 535 following identity:

$$
\begin{aligned}
& F_{D}^{(n)}\left[a, b_{1}, \cdots, b_{n}, c, x_{1}, \cdots, x_{n}\right]=\prod_{i=1}^{n}\left(1-x_{i}\right)^{-b_{i}} \\
& F_{D}^{(n)}\left[a, b_{1}, \cdots, b_{n}, c, \frac{x_{1}}{x_{1}-1}, \cdots, \frac{x_{n}}{x_{n}-1}\right],
\end{aligned}
$$

we arrive at:

$$
\begin{aligned}
& \operatorname{EVM}_{\eta-\mu, i . n . i . d}=K_{1} \theta_{1}^{\sum_{i=1}^{2 N} \alpha_{i}-0.5} \Gamma\left(\sum_{i=1}^{2 N} \alpha_{i}-0.5\right) \prod_{i=2}^{2 N}\left(\frac{\theta_{1}}{\theta_{i}}\right)^{-\alpha_{i}} \\
& \times F_{D}^{(2 N-1)}\left(0.5, \alpha_{2}, \cdots, \alpha_{2 N} ; \sum_{i=1}^{2 N} \alpha_{i} ; 1-\frac{\theta_{2}}{\theta_{1}}, \cdots, 1-\frac{\theta_{2 N}}{\theta_{1}}\right) .
\end{aligned}
$$

Substituting the value of $K_{1}$, the $\mathrm{EVM}_{\eta-\mu, i . n . i . d}$ expression can 538 


$$
\begin{aligned}
& \text { be simplified to } \\
& \mathrm{EVM}_{\eta-\mu, \text { i.n.i.d }}=\frac{1}{\sqrt{\theta_{1} S N R_{S I M O}}} \frac{\Gamma\left(\sum_{i=1}^{2 N} \alpha_{i}-0.5\right)}{\Gamma\left(\sum_{i=1}^{2 N} \alpha_{i}\right)} \\
& \times F_{D}^{(2 N-1)}\left(0.5, \alpha_{2}, \cdots, \alpha_{2 N} ; \sum_{i=1}^{2 N} \alpha_{i} ; 1-\frac{\theta_{2}}{\theta_{1}}, \cdots, 1-\frac{\theta_{2 N}}{\theta_{1}}\right) .
\end{aligned}
$$

Substituting the value of $\theta_{i}, \alpha_{i}$ and $\bar{z}=1 / N$ into (45), we obtain the final expression of $\mathrm{EVM}_{\eta-\mu, \text { i.n.i.d }}$ given in (14).

\section{APPENDIX B}

The underlying philosophy in this derivation is similar to that of an $\eta-\mu$ SIMO channel with i.n.i.d branches. We now consider the scenario studies in this paper, where $Z$ is the sum of $N$ correlated and identically distributed $\eta-\mu \mathrm{RVs} X_{k}$ having distribution parameters $\left\{\eta_{k}, \mu_{c}\right\}$. Note that all the $X_{k}$ s have the same $\mu_{c}$ but different $\eta_{k}$. As discussed in the first paragraph of Section III-A, an $\eta-\mu$ random variable may be expressed as the sum of two independent Gamma distributed RVs. It has been discussed in [28] that each $X_{k}$ may be expressed as

$$
X_{k}=P_{k}+Q_{k},
$$

where $P_{k} \sim \mathcal{G}\left(\mu_{c}, \theta_{2 k-1}\right)$, and $Q_{k} \sim \mathcal{G}\left(\mu_{c}, \theta_{2 k}\right)$ with $\theta_{2 k-1}=$ $\frac{\bar{x}_{k}}{2 \mu_{c}\left(h_{k}+H_{k}\right)}$ and $\theta_{2 k}=\frac{\bar{x}_{k}}{2 \mu_{c}\left(h_{k}-H_{k}\right)}$. Similar to Section III-A, $\bar{x}_{k}=1 / N$, while $h_{k}$ and $H_{k}$ are given by (4). Note that the correlation among the different $X_{k}$ s results in a correlation among the different $P_{k}$ s and among the $Q_{k}$ s, but there is no correlation between the $P_{k}$ s and $Q_{k}$ s. If $\rho_{i j}^{x x}$ is the correlation between $X_{i}=P_{i}+Q_{i}$ and $X_{j}=P_{j}+Q_{j}$, while $\rho_{i j}^{p p}$ and $\rho_{i j}^{q q}$ is the correlation between $\left\{P_{i}, P_{j}\right\}$ and $\left\{Q_{i}, Q_{j}\right\}$, respectively then we have (47), shown at the bottom of the page.

In our study $Z$ is the sum of $N$ correlated and identically distributed $\eta-\mu$ RVs $X_{k}$. Employing (46), we may state that $Z$ is the sum of $2 N$ correlated and non-identically distributed Gamma distributed RVs $M_{i}$, namely $\left\{M_{1}=P_{1}, M_{2}=\right.$ $\left.Q_{1}, M_{3}=P_{2}, M_{4}=Q_{2} \ldots, M_{2 N-1}=P_{N}, M_{2 N}=Q_{N}\right\}$.

The pdf of the sum of $N$ correlated $\eta-\mu$ math RVs is given by [16], [29], [33]

$$
\begin{aligned}
& F_{Z, \eta-\mu, c o r r}(z)=\frac{z^{2 N \mu_{c}-1}}{\operatorname{det}(\mathbf{A})^{\mu_{c} \Gamma\left(2 N \mu_{c}\right)}} \\
& \quad \times \Phi_{2}^{(2 N)}\left(\mu_{c}, \cdots, \mu_{c} ; 2 N \mu_{c} ; \frac{-z}{\hat{\theta}_{1}}, \cdots, \frac{-z}{\hat{\theta}_{2 N}}\right),
\end{aligned}
$$

where $\hat{\theta}_{i}$ is the eigen values of $\mathbf{A}=\mathbf{D C}$ with $\mathbf{D}$ being a diago- 568 nal matrix with entries $\theta_{i}$ and $\operatorname{det}(\mathbf{A})=\prod_{i=1}^{N} \hat{\theta}_{i}$ is the determinant 569 of the matrix A. Here, $\mathbf{C}$ is the symmetric positive definite 570 (s.p.d) matrix and is given in (49), shown at the bottom of the 571 page. where $\rho_{i j}^{m m}$ denotes the correlation coefficient between 572 $M_{i}$ and $M_{j}$, and is given by,

$$
\rho_{i j}^{m m}=\rho_{j i}^{m m}=\frac{\operatorname{cov}\left(M_{i}, M_{j}\right)}{\sqrt{\operatorname{var}\left(M_{i}\right) \operatorname{var}\left(M_{j}\right)}}, 0 \leq \rho_{i j} \leq 1,
$$

with $\operatorname{cov}\left(M_{i}, M_{j}\right)$ being the covariance between $M_{i}$ and $M_{j} .574$ Note that the alternate zeros are a consequence of $P_{k} \mathrm{~s}$ and $Q_{k} \mathrm{~s} \quad 575$ being independent.

Just as in (36), the EVM of a SIMO channel encountering 577 correlated $\eta-\mu$ fading and employing MRC reception is defined 578 as follows:

$$
\operatorname{EVM}_{\eta-\mu, \text { corr }}=\int_{0}^{\infty} \operatorname{EVM}(z) f_{Z, \eta-\mu, c o r r}(z) \mathrm{d} z,
$$

The functional form of the pdf of the sum of correlated gamma 580 $\mathrm{RVs}$ is similar to that of the sum of i.n.i.d. $\eta-\mu \mathrm{RVs}$, as given 581 in (13). Hence the EVM expression in (51) may be readily 582 simplified to:

$$
\begin{aligned}
& \operatorname{EVM}_{\eta-\mu, c o r r}=\frac{1}{\sqrt{\hat{\theta}_{1} S N R_{S I M O}}} \frac{\Gamma\left(2 N \mu_{c}-0.5\right)}{\Gamma\left(2 N \mu_{c}\right)} \\
& \times F_{D}^{(2 N-1)}\left(0.5, \mu_{c}, \cdots, \mu_{c} ; 2 N \mu_{c} ;\left(1-\frac{\hat{\theta}_{2}}{\hat{\theta}_{1}}\right), \cdots\left(1-\frac{\hat{\theta}_{2 N}}{\hat{\theta}_{1}}\right)\right)
\end{aligned}
$$

Note that $\hat{\theta}_{i}$ is the eigen values of $\mathbf{A}=\mathbf{D C}$ with $\mathbf{D}$ being a 584 diagonal matrix with entries $\theta_{i}$ and $\mathbf{C}$ is the symmetric positive 585 definite (s.p.d) covariance matrix defined in (49).

$$
\rho_{i j}^{x x}=\frac{\rho_{i j}^{p p} \sqrt{\operatorname{var}\left(P_{i}\right) \operatorname{var}\left(P_{j}\right)}+\rho_{i j}^{q q} \sqrt{\operatorname{var}\left(Q_{i}\right) \operatorname{var}\left(Q_{j}\right)}}{\sqrt{\operatorname{var}\left(P_{i}\right) \operatorname{var}\left(P_{j}\right)+\operatorname{var}\left(Q_{i}\right) \operatorname{var}\left(Q_{j}\right)+\operatorname{var}\left(P_{i}\right) \operatorname{var}\left(Q_{j}\right)+\operatorname{var}\left(P_{j}\right) \operatorname{var}\left(Q_{i}\right)}}
$$

$$
\mathbf{C}=\left[\begin{array}{ccccccccc}
1 & 0 & \sqrt{\rho_{12}^{p p}} & 0 & \sqrt{\rho_{13}^{p p}} & 0 & \cdots & \sqrt{\rho_{1 N}^{p p}} & 0 \\
0 & 1 & 0 & \sqrt{\rho_{12}^{q q}} & 0 & \sqrt{\rho_{13}^{q q}} & \cdots & 0 & \sqrt{\rho_{1 N}^{q q}} \\
\sqrt{\rho_{21}^{p p}} & 0 & 1 & 0 & \sqrt{\rho_{23}^{p p}} & 0 & \cdots & \sqrt{\rho_{2 N}^{p p}} & 0 \\
\vdots & \vdots & \vdots & \vdots & \vdots & \vdots & \vdots & \vdots & \vdots \\
0 & \sqrt{\rho_{N 1}^{q q}} & 0 & \sqrt{\rho_{N 2}^{q q}} & 0 & \sqrt{\rho_{N 3}^{q q}} & \cdots & 0 & 1
\end{array}\right] .
$$


$589 \beta_{i}=\frac{\bar{x}_{i}}{\mu_{i}\left(1+\kappa_{i}\right)}$ and $\delta_{i}=\frac{\left(\mu_{i} \kappa_{i}+m_{i}\right) \bar{x}_{i}}{\mu_{i}\left(1+\kappa_{i}\right) m_{i}}$, we can rewrite the pdf $590 f_{Z, \kappa-\mu s h}(z)$ as

$$
\begin{aligned}
& f_{Z, \kappa-\mu s h}(z)=\left(\prod_{i=1}^{N} \frac{1}{\beta_{i}^{\mu_{i}-m_{i}} \delta_{i}^{m_{i}}}\right) \frac{z^{\sum^{i=1}} \mu_{i}-1}{\Gamma\left(\sum_{i=1}^{N} \mu_{i}\right)} \\
& \times \Phi_{2}^{(2 N)}\left(\mu_{1}-m_{1}, \cdots, \mu_{N}-m_{N}, m_{1} \cdots, m_{N} ;\right. \\
& \left.\sum_{i=1}^{N} \mu_{i} ;-\frac{z}{\beta_{1}}, \cdots,-\frac{z}{\beta_{N}},-\frac{z}{\delta_{1}}, \cdots, \frac{z}{\delta_{N}}\right) .
\end{aligned}
$$

591 The EVM of $\kappa-\mu$ shadow fading SIMO channel with i.n.i.d 592

branches is defined as follows [24]:

$$
\operatorname{EVM}_{\kappa-\mu s h, i . n . i . d}=\int_{0}^{\infty} \operatorname{EVM}(z) f_{Z, \kappa-\mu s h}(z) \mathrm{d} z .
$$

593 Note that the functional form of the pdf of the sum of $\kappa-\mu$ shad-

594

595

596 owed RVs is similar to that of the sum of $\eta-\mu \mathrm{RVs}$, as given in (13). Hence the EVM of the $\kappa-\mu$ shadowed fading SIMO channel with i.n.i.d branches may be expressed as follows:

$$
\begin{aligned}
& \operatorname{EVM}_{\kappa-\mu s h, i . n . i . d}=\frac{1}{\sqrt{\beta_{1} S N R_{S I M O}}} \frac{\Gamma\left(\sum_{i=1}^{N} \mu_{i}-0.5\right)}{\Gamma\left(\sum_{i=1}^{N} \mu_{i}\right)} \\
& \times F_{D}^{(2 N-1)}\left(0.5, \mu_{2}-m_{2}, \cdots, \mu_{2}-m_{2}, m_{1}, \cdots m_{N} ;\right. \\
& \left.\sum_{i=1}^{N} \mu_{i} ; 1-\frac{\beta_{2}}{\beta_{1}}, \cdots, 1-\frac{\beta_{N}}{\beta_{1}}, 1-\frac{\delta_{1}}{\beta_{1}} \cdots 1-\frac{\delta_{N}}{\beta_{1}}\right) .
\end{aligned}
$$

597
Substituting the value of $\beta_{i}$ and $\delta_{i}$ and $\bar{x}_{i}=1 / N \forall i$ into (55), we obtain the final expression of $\mathrm{EVM}_{\kappa}-\mu$ sh,i.n.i.d, which is given in (27).

\section{REFERENCES}

[1] J. Zhang, Z. Tan, H. Wang, Q. Huang, and L. Hanzo, "The effective throughput of MISO systems over $\kappa-\mu$ fading channels," IEEE Trans. Veh. Technol., vol. 63, no. 2, pp. 943-947, Feb. 2014.

[2] V. Aalo, T. Piboongungon, and G. Efthymoglou, "Another look at the performance of MRC schemes in Nakagami-M fading channels with arbitrary parameters," IEEE Trans. Commun., vol. 53, no. 12, pp. 2002-2005, Dec. 2005.

[3] D. Chen, L.-L. Yang, and L. Hanzo, "Multi-hop diversity aided multi-hop communications: A cumulative distribution function aware approach," IEEE Trans. Commun., vol. 61, no. 11, pp. 4486-4499, Nov. 2013.

[4] L. Hanzo, S. X. Ng, T. Keller, and W. Webb, Quadrature Amplitude Modulation, 2nd ed. Hoboken, NJ, USA: Wiley, 2004.

[5] R. Vaughan, N. Scott, and D. White, "Eight hints for making and interpreting EVM measurements," Agilent Application Note, May 2005, pp. 1-12.

[6] R. Liu, Y. Li, H. Chen, and Z. Wang, "EVM estimation by analyzing transmitter imperfections mathematically and graphically," Analog Integr. Circuits Signal Process., vol. 48, no. 3, pp. 257-262, Jan. 2014.

[7] A. Georgiadis, "Gain, phase imbalance, and phase noise effects on error vector magnitude," IEEE Trans. Veh. Technol., vol. 53, no. 2, pp. 443449, Mar. 2004.
[8] R. Shafik, S. Rahman, R. Islam, and N. Ashraf, "On the error vector mag- 622 nitude as a performance metric and comparative analysis," in Proc. Int. 623 Conf. Emerg. Technol. (ICET), Nov 2006, pp. 27-31. 624

[9] D. Tse and P. Viswanath, Fundamentals of Wireless Communication. 625 Cambridge, U.K.: Cambridge Univ. Press, 2005.

[10] M. D. Yacoub, "The $\kappa-\mu$ distribution and the $\eta$ - $\mu$ distribution," IEEE 627 Antennas Propag. Mag., vol. 49, no. 1, pp. 68-81, Feb. 2007.

[11] J. Paris, "Statistical characterization of $\kappa-\mu$ shadowed fading," IEEE 629 Trans. Veh. Technol., vol. 63, no. 2, pp. 518-526, Feb. 2014.

[12] M. Arti, "Channel estimation and detection in hybrid satellite- 631 terrestrial communication systems," IEEE Trans. Veh. Technol., 2015, 632 http://ieeexplore.ieee.org/xpl/login.jsp?tp=\&arnumber=7156170, to be 633 published.

[13] M. Arti and M. Bhatnagar, "Beamforming and combining in hybrid 635 satellite-terrestrial cooperative systems," IEEE Commun. Lett., vol. 18, 636 no. 3, pp. 483-486, Mar. 2014.

[14] M. Arti and M. Bhatnagar, "Two-way mobile satellite relaying: A beam- 638 forming and combining based approach," IEEE Commun. Lett., vol. 18, 639 no. 7, pp. 1187-1190, Jul. 2014.

[15] M. Arti, "Imperfect CSI based maximal ratio combining in shadowed- 641 rician fading land mobile satellite channels," in Proc. 21st Nat. Conf. 642 Commun. (NCC), Feb. 2015, pp. 1-6.

[16] M. Bhatnagar, "On the sum of correlated squared $\kappa$ - $\mu$ shadowed ran- 644 dom variables and its application to performance analysis of MRC," IEEE 645 Trans. Veh. Technol., vol. 64, no. 6, pp. 2678-2684, Jun. 2015.

[17] J. F. Paris, "Outage probability in $\eta-\mu / \eta-\mu$ and $\kappa-\mu / \eta-\mu$ interference- 647 limited scenarios," IEEE Trans. Commun., vol. 61, no. 1, pp. 335-343, 648 Jan. 2013.

[18] D. da Costa and M. Yacoub, "Average channel capacity for generalized 650 fading scenarios," IEEE Commun. Lett., vol. 11, no. 12, pp. 949-951, 651 Dec. 2007.

[19] S. Kumar, G. Chandrasekaran, and S. Kalyani, "Analysis of outage prob- 653 ability and capacity for $\kappa-\mu / \eta-\mu$ faded channel," IEEE Commun. Lett., 654 vol. 19, no. 2, pp. 211-214, Feb. 2015

[20] L. Hanzo, M. El-Hajjar, and O. Alamri, "Near-capacity wireless 6 transceivers and cooperative communications in the MIMO era: 657 Evolution of standards, waveform design, and future perspectives," Proc. 658 IEEE, vol. 99, no. 8, pp. 1343-1385, Aug. 2011.

[21] M. Bhatnagar and M. Arti "On the closed-form performance analysis 660 of maximal ratio combining in shadowed-rician fading LMS channels," 661 IEEE Commun. Lett., vol. 18, no. 1, pp. 54-57, Jan. 2014.

[22] K. Gharaibeh, K. Gard, and M. Steer, "Accurate estimation of digital 663 communication system metrics-SNR, EVM and $\rho$; in a nonlinear ampli- 664 fier environment," in Proc. ARFTG Microw. Meas. Conf., Dec. 2004, 665 pp. 41-44.

[23] R. Shafik, S. Rahman, and R. Islam, "On the extended relationships 667 among EVM, BER and SNR as performance metrics," in Proc. Int. Conf. 668 Elect. Comput. Eng. (ICECE), Dec. 2006, pp. 408-411.

[24] H. Mahmoud and H. Arslan, "Error vector magnitude to SNR conver- 670 sion for nondata-aided receivers," IEEE Trans. Wireless Commun., vol. 8, 671 no. 5, pp. 2694-2704, May 2009.

[25] N. Ermolova and O. Tirkkonen, "Bivariate $\eta$ - $\mu$ fading distribution with 673 application to analysis of diversity systems," IEEE Trans. Wireless 674 Commun., vol. 10, no. 10, pp. 3158-3162, Oct. 2011.

[26] N. Y. Ermolova, "Moment generating functions of the generalized $\eta-\mu \quad 676$ and $\kappa-\mu$ distributions and their applications to performance evaluations of 677 communication systems," IEEE Commun. Lett., vol. 12, no. 7, pp. 502- 678 504, Jul. 2008.

[27] K. Peppas, F. Lazarakis, A. Alexandridis, and K. Dangakis "Error per- 680 formance of digital modulation schemes with MRC diversity reception 681 over $\eta$ - $\mu$ fading channels," IEEE Trans. Wireless Commun., vol. 8, no. 10, 682 pp. 4974-4980, Oct. 2009.

[28] N. Y. Ermolova and O. Tirkkonen, "Bivariate $\eta$ - $\mu$ fading distribution with application to analysis of diversity systems," IEEE Trans. Wireless Commun., vol. 10, no. 10, pp. 3158-3162, Oct. 2011.

[29] S. Kalyani and R. Karthik, "The asymptotic distribution of maxima of independent and identically distributed sums of correlated or nonidentical gamma random variables and its applications," IEEE Trans. Commun., vol. 60, no. 9, pp. 2747-2758, Sep. 2012.

[30] H. Exton, Multiple Hypergeometric Functions and Applications. New York, NY, USA: Halsted Press, Ellis Horwood, 1976.

[31] F. G. Tricomi and A. Erdlyi, "The asymptotic expansion of a ratio of 693 gamma functions," Pac. J. Math., vol. 1, no. 1, pp. 133-142, 1951.

32] N. M. Temme, "Large parameter cases of the gauss hypergeometric 695 function," J. Comput. Appl. Math., vol. 153, no. 1, pp. 441-462, 2003.696

[33] S. Kumar and S. Kalyani, "Coverage probability and rate for $\eta-\mu / \kappa-\mu \quad 697$ fading channels in interference-limited scenarios," IEEE Trans. Wireless 698 Commun., vol. 14, no. 11, pp. 6082-6096, Nov. 2015.

3
4
5
7
8
9
0
1
2
3
4
5
6
7
8
9
0
1
2
3
4
5
6
7
8
9

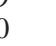

,




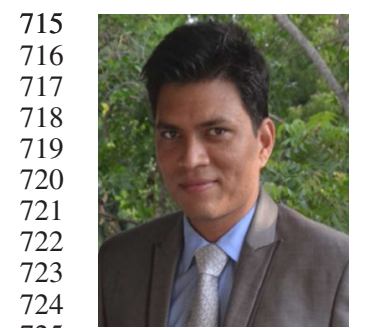

725

726

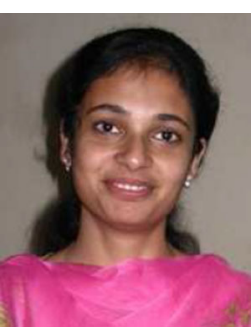

Sheetal Kalyani received the B.E. degree in electronics and communication engineering from Sardar Patel University, Gujarat, India, in 2002, and the Ph.D. degree in electrical engineering from the Indian Institute of Technology (IIT) Madras, Chennai, India, in 2008. She was a Senior Research Engineer with the Centre of Excellence in Wireless Technology, Chennai, India, from 2008 to 2012. She is is currently an Assistant Professor with the Department of Electrical Engineering, IIT Madras.

Her research interests include HetNets, extreme value theory, hypergeometric functions, generalized fading models, and statistical learning algorithms for prediction in wireless networks.

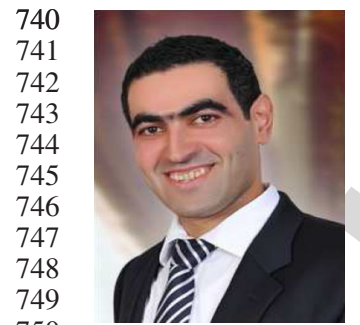

Mohammed El-Hajjar received the B.Eng. degree in electrical engineering from the American University of Beirut, Lebanon, in 2004, and the M.Sc. degree in radio frequency communication systems and the $\mathrm{Ph} . \mathrm{D}$. degree in wireless communications from the University of Southampton, Southampton, U.K., in 2005 and 2008, respectively. Following the Ph.D., he joined Imagination Technologies as a Design Engineer, where he worked on designing and developing Imagination's multistandard communications platform, which resulted in three patents. Since January 2012, he has been a Lecturer with the Southampton Wireless Research Group, School of Electronics and Computer Science, University of Southampton, Southampton, U.K. He has authored a Wiley-IEEE book and more than 60 journal and international conference papers. His research interests include the development of intelligent communications systems including energy-efficient transceiver design, multifunctional MIMO, millimetre wave communications, and radio over fibre systems.

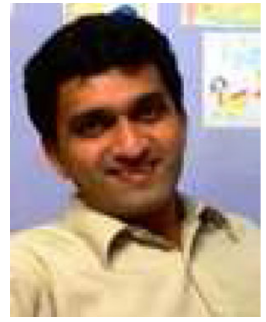

K. Giridhar received the B.Sc. degree in applied sci- 758 ences from PSG College of Technology, Coimbatore, 759 India, the M.E. degree in electrical communications 760 from Indian Institute of Science, Bangalore, India, 761 and the Ph.D. degree in electrical engineering from 762 the University of California, Santa Barbara, Santa 763 Barbara, CA, USA. Between 1989 and 1990, he 764 was a Member of Research Staff with CRL, Bharat 765 Electronics, Bangalore, India, and between 1993 and 766 1994, was a Research Affiliate in electrical engineer- 767 ing with Stanford University, Stanford, CA, USA. 768 Since 1994, he has been with the Department of Electrical Engineering, IIT 769 Madras (IITM), Chennai, India.

He serves as a Consultant to many Telecom \& VLSI companies in India, 771 and was on a Sabbatical from 2004 to 2005 with Beceem Communications. 772 He has been a Visiting Faculty at Sri Sathya Sai Institute of Higher Learning, 773 Prasanthi Nilayam, Anantapur, India, and Stanford University. His research 774 interests include adaptive signal processing and wireless communications sys- 775 tems, with an emphasis on various transceiver algorithms, custom air-interface 776 design for strategic applications, and performance analysis of mobile broadband 777 wireless networks including HetNets.

$\mathrm{He}$ is a Member of the Telecommunications and Computer Networks (TeNeT) Group at IITM. He actively collaborates with the Center of Excellence in Wireless Technology on MIMO-OFDM broadband access research, resulting in several contributions to IEEE $802.16 \mathrm{~m}$, and currently on proposals to LTE-A and $5 \mathrm{G}$ forums.


Lajos Hanzo (M'91-\&SM'92-F'04) received the degree in electronics in 1976 and the doctorate degree in 1983. During his 40-year career in telecommunications, he has held various research and academic posts in Hungary, Germany, and the U.K. Since 1986 he has been with the School of Electronics and Computer Science, University of Southampton, U.K. where he holds the chair in telecommunications. He has successfully supervised about $100 \mathrm{Ph} . \mathrm{D}$. students, co-authored 20 John Wiley/IEEE Press books on mobile radio communications totalling in excess of 10,000 pages, published over 1500 research entries at IEEE Xplore, acted both as the TPC and the General Chair of IEEE conferences, presented keynote lec tures, and has been awarded a number of distinctions. Currently, he is directin a 60 -strong academic research team, working on a range of research projects in the field of wireless multimedia communications sponsored by industry, the Engineering, and Physical Sciences Research Council (EPSRC), U.K., the European Research Council's Advanced Fellow Grant, and the Royal Society's Wolfson Research Merit Award. His research is funded by the European Research Council's Senior Research Fellow Grant. Lajos has over 23,000 citations. He is an enthusiastic supporter of industrial and academic liaison and offers a range of industrial courses. He is a Fellow of Royal Academy of 805 Engineering, the Institution of Engineering and Technology, the EURASIP, and 806 DSc. He is also a Governor of the IEEE VTS. From 2008 to 2012, he was the 807 Editor-in-Chief of the IEEE Press and also a Chaired Professor at Tsinghua 808 University, Beijing, China. In 2009, he was awarded an Honorary Doctorate 809 by the Technical University of Budapest, while in 2015 by the University of 810 Edinburgh.
784 785 786 787 807 . 


\title{
Error Vector Magnitude Analysis of Fading SIMO Channels Relying on MRC Reception
}

\author{
Varghese Antony Thomas, Suman Kumar, Sheetal Kalyani, Mohammed El-Hajjar, K. Giridhar, \\ and Lajos Hanzo, Fellow, IEEE
}

\begin{abstract}
5 Abstract-We analytically characterize the data-aided error 6 vector magnitude (EVM) performance of a single-input multiple7 output (SIMO) communication system relying on maximal ratio 8 combining (MRC) having either independent or correlated 9 branches that are nonidentically distributed. In particular, exact 10 closed form expressions are derived for the EVM in $\eta-\mu$ fading and $1 \kappa-\mu$ shadowed fading channels and these expressions are validated 12 by simulations. The derived expressions are expressed in terms 13 of Lauricella's function of the fourth kind $F_{D}^{(N)}($.$) , which can$ 14 be easily computed. Furthermore, we have simplified the derived 15 expressions for various special cases such as independent and iden16 tically distributed branches, Rayleigh fading, Nakagami- $m$ fading, 17 and $\kappa-\mu$ fading. Additionally, a parametric study of the EVM 18 performance of the wireless system is presented.
\end{abstract}

Index Terms-Error Vector Magnitude, maximal ratio combining, $\eta-\mu$ fading, $\kappa-\mu$ fading, SIMO.

\section{INTRODUCTION}

C ONFORMITY with the wireless communication performance standards is an absolute necessity, when designing communication systems. Traditional approaches of quantifying a communication system's performance includes the calculation of classic metrics such as the Bit Error Ratio (BER), the throughput and the outage probability [1]-[4]. However, an alternative metric that is becoming increasingly popular is the Error Vector Magnitude (EVM) [5].

EVM as a performance metric offers several advantages. Firstly, it facilitates the identification of the specific types of degradations encountered, in addition to their particular sources in a transmission link [5]. Some of these degradations are the Inphase-Quadrature Phase (IQ) imbalance, the Local Oscillator's (LO) phase noise, carrier leakage, nonlinearity and the LO's frequency error [6], [7]. Secondly, the EVM is a symbol-level performance metric unlike the BER, which is a

Manuscript received July 21, 2015; revised December 12015 and February 8, 2016; accepted February 12, 2016. The financial support of the EPSRC projects EP/Noo4558/1 and EP/L018659/1, as well as of the European Research Council's Advanced Fellow Grant under the Beam-Me-Up project and of the Royal Society's Wolfson Research Merit Award is gratefully acknowledged. V. A. Thomas and S. Kumar are co-first authors. The associate editor coordinating the review of this paper and approving it for publication was T. A. Tsiftsis.

V. A. Thomas, M. El-Hajjar, and L. Hanzo are with the School of Electronics and Computer Science, University of Southampton, Southampton SO17 1BJ, U.K. (e-mail: lh@ecs.soton.ac.uk).

S. Kumar, S. Kalyani, and K. Giridhar are with the Department of Electrical Engineering, Indian Institute of Technology Madras, Chennai 600036, India.

Color versions of one or more of the figures in this paper are available online at http://ieeexplore.iee.org.

Digital Object Identifier 10.1109/TCOMM.2016.2530797 bit-level performance metric. Hence, EVM is more convenient for Symbol Error Rate (SER) based scenarios where multiple modulation schemes are employed, as in adaptive modulation [8]. Thirdly, it may be employed by a communication system designer for ensuring conformity with wireless standards, because EVM-based specifications have already become a part of the Wideband Code Division Multiple Access (W-CDMA) and IEEE 802.11 family of Wireless Local Area Network (WLAN) standards [5], [8]. Fourthly, in experimental studies the channel model used is often a proprietary channel, for which no closed form expressions are available either for the BER or for the EVM. In these studies, the designer has to characterize the system by transmitting and receiving bits, where the BER calculation relying on the Monte Carlo approach has a long computation time, especially at low BERs. By contrast, the EVM can be readily evaluated by transmitting fewer symbols, as compared to the BER. Hence, characterizing the performance using EVM is preferred. However, in contrast to the classic BER formulae, the current literature does not provide closed form expressions of the EVM of several important channel scenarios. Hence provides closed-form expressions for some of these important channel scenarios and partially fills this gap in the open literature. We have now added the following text to the discussions in the introduction section (please see page 2 of the revised manuscript). Moreover, EVM is easier to employ than BER as a performance metric in systems, where the transmitter requires feedback regarding the link's performance for making choices such as which adaptive modulation mode or channel coding rate to rely on. This is because employing BER would require the received signal to go through the entire receive chain before the feedback can be generated, while computation of the EVM using the received symbols would be quicker. Thus, employing EVM would be a better choice for providing real-time feedback.

In an optimized system the major source of degradation is the channel's fading [4], [9]. However, in a realistic system a range of degradations mentioned in [5] are imposed, which would play a detrimental role. Employing EVM would help the designer identify these impairments at a glance and hence to mitigate them. Mitigating the effects of these distortions would require the EVM of the the best-case scenario, where the EVM is predominantly or purely decided by the wireless channel's fading as well as by the ubiquitous receiver-noise, and not by other impairments, such as non-linear distortions and synchronization errors, etc. Hence in this paper we aim for providing the designer with closed form expressions for 
determining this best-case target EVM. Numerous models have been employed in the literature for simulating a wireless channel [4]. Some of these models have been used for several years, including the AWGN, Rayleigh, Rician and the Nakagami-m as well as the Nakagami-q faded channels. On the other hand, recent studies are increasingly favouring the state-of-the-art $\eta-\mu$ and $\kappa-\mu$ shadowed fading channels [10], [11], because they represent all-encompassing generalizations, with the classical channels being their special cases. For example, the $\eta-\mu$ distribution includes the Nakagami-q (Hoyt), the Nakagami-m, the Rayleigh and the One-Sided Gaussian distribution as special cases. The $\kappa-\mu$ distribution includes the Nakagami-n (Rice), the Nakagami-m, the Rayleigh, and the One-Sided Gaussian distribution as special cases. The $\kappa-\mu$ shadowed distribution includes $\kappa-\mu$ and Rician shadowed distribution as special cases. Moreover, they match the experimentally measured mobile radio propagation statistics better than the other channel models [10]. The $\kappa-\mu$ shadowed fading is useful for modelling the satellite links. A simplified model for $\kappa-\mu$ fading is the shadowed-Rician fading, which has been employed for modelling the satellite links [12]-[15].

The BER, outage probability and capacity are some commonly employed performance metrics, which have been quantified for $\eta-\mu$ and $\kappa-\mu$ shadowed fading ${ }^{1}$ channels in [16]-[19] and in the references therein. On the other hand, there is a dearth of studies that focus on the quantification of the achievable EVM for these wireless channels. Moreover, there are no studies that characterize the EVM performance for the commonly employed wireless technique of receive antenna diversity [20]. Note that a performance analysis of maximal ratio combining based receive antenna diversity was provided in [21] for the case of the shadowed-Rician fading land mobile satellite channels. Employing multiple receive antennas provides a diversity gain [20], where the link between the transmit antenna and each receive antenna is referred to as a single branch of the Single Input Multiple Output (SIMO) channel. The fading coefficients of the different branches may be independently distributed or correlated, where the branches in these scenearios are referred to as being independent or correlated, respectively. Additionally, they may have the same or different probability distribution parameters, where the branches in these scenearios are referred to as being identically or non-identically distributed, respectively. It must be noted that there is some literature on the EVM performance of the classical AWGN and Rayleigh channels for the scenario of a single receive antenna, though these are limited to only a couple of research papers. The seminal effort was made in this direction in [22], while [23] formulates the attainable EVM in an AWGN scenario. This study was extended in [24] to the scenario of non data-aided receivers communicating over both $\mathrm{AWGN}$ as well as Rayleigh fading channels.

A designer can compute the expected BER for various fading channels using well established formulae from the existing literature. Thus, designers have a benchmark with which they

\footnotetext{
${ }^{1}$ The probability distribution function (pdf) of the sum of the squared $\kappa-\mu$ shadowed random variables with independent and correlated shadowing components are derived in [11] and [16], respectively. Note that the pdf derived in [11] is a special case of the pdf derived in [16].
}

can compare the experimental results, when using BER as a 138 performance metric. However, there are no such equivalent the- 139 oretical formulae for EVM. Hence, through this paper we aim 140 to provide a theoretical benchmark for the EVM performance 141 that the designer can expect in the wireless channels. 142

Against this background, the novel contributions of this 143 paper may be summarised as follows:

1) We derive exact closed form expressions for the dataaided $\mathrm{EVM}^{2}$ performance of a SIMO wireless system employing the $\eta-\mu$ and $\kappa-\mu$ shadowed fading channels and a Maximal Ratio Combining (MRC) receiver. Our expressions are derived for independent and non identically distributed branches. These results are then validated by simulations ${ }^{3}$.

2) We also study the effect of correlated fading channels in the above-mentioned wireless system and formulate the EVM for these scenarios.

3) The expressions derived are then further simplified for 155 various special cases, such as independent and identically 156 distributed branches, the Rayleigh, the Nakagami and the 157 $\kappa-\mu$ fading.

4) The impact of the various channel parameters such as $\eta, 159$ $\mu, \kappa$ and that of the number of receive antennas $N$ on 160 the EVM performance is studied along with the attainable 161 performance limits.

Our paper is organized as follows. In Section II, we present 163 the background necessary for understanding this study, which 164 includes discussions on the SIMO $\eta-\mu$ and $\kappa-\mu$ shadowed 165 channel models in Section II-A and on EVM in Section II-B. 166 Subsequently, we present our analytical characterization of the 167 EVM performance for a SIMO wireless system in Section III, 168 while in Section IV we provide our simulation results. Finally, 169 we offer our conclusions in Section V.

\section{BACKGROUND INFORMATION}

\section{A. SIMO $\eta-\mu$ and $\kappa-\mu$ Shadowed Channel Models}

For the case of a SIMO wireless channel having $N$ receive 173 antennas, the channel model is as follows [4]:

$$
\hat{\mathbf{y}}=\mathbf{h} s+\mathbf{n},
$$

where $s$ is the transmitted symbol and

$$
\begin{aligned}
\hat{\mathbf{y}} & =\left[\begin{array}{llll}
\hat{y}_{1} & \hat{y}_{2} & \cdots & \hat{y}_{N}
\end{array}\right]^{T} \\
\mathbf{h} & =\left[\begin{array}{llll}
a_{1} e^{j \theta_{1}} & a_{2} e^{j \theta_{2}} & \cdots & a_{N} e^{j \theta_{N}}
\end{array}\right]^{T} \\
\mathbf{n} & =\left[\begin{array}{llll}
n_{1} & n_{2} & \cdots & n_{N}
\end{array}\right]^{T} .
\end{aligned}
$$

Here $\hat{y}_{k}$ is the symbol received by the $k^{t h}$ receive antenna after 176 being subjected to the multiplicative fading of $a_{k} e^{j \theta_{k}}$ and to 177 corruption by the additive noise of $n_{k}$. In the above discussions 178 $a_{k}, \theta_{k}$ and $n_{k}$ are random variables (RVs), whose pdf has to 179 be experimentally characterized. Typically the noise is mod- 180 elled by a zero-mean Gaussian distribution, while the phase of 181

\footnotetext{
${ }^{2}$ Note that data-aided EVM refers to the EVM obtained using data-aided receivers, i.e receivers which have exact knowledge of the transmitted bits.

${ }^{3}$ Please note that if any other detector than the MRC is used, then the EVM analysis will change significantly.
} 
the fading co-efficient is assumed to have a uniform distribution within $[0,2 \pi][4]$. However, modelling the distribution of $a_{k}$ or alternatively that of $X_{k} \propto a_{k}^{2}$ is much more challenging due to its heavy dependence on the exact nature of the wireless channel. Note that $X_{k}$ is referred to as the fading power.

Recently $\eta-\mu$ and $\kappa-\mu$ shadowed pdfs were proposed in [10] and [11], respectively. Mathematically, the $\eta-\mu$ fading power (or fading attenuation) pdf is expressed as follows for each $X_{k}$ [10], [25]:

$$
\begin{aligned}
f_{X_{k}, \eta-\mu}(x)= & \frac{2 \sqrt{\pi} \mu_{k}^{\mu_{k}+\frac{1}{2}} h_{k}^{\mu_{k}}}{\Gamma\left(\mu_{k}\right) H_{k}^{\mu_{k}-\frac{1}{2}} \bar{x}_{k}^{\mu_{k}+\frac{1}{2}}} x^{\mu_{k}-\frac{1}{2}} e^{\frac{-2 \mu_{k} h_{k} x}{\bar{x}_{k}}} . \\
& \times I_{\mu_{k}-\frac{1}{2}}\left(\frac{2 \mu_{k} H_{k} x}{\bar{x}_{k}}\right),
\end{aligned}
$$

where the modified Bessel function of the first kind of order $b$ is represented by $I_{b}($.$) and the Gamma function is denoted by$ $\Gamma$ (.) [10]. Here we have $\mu_{k}=\frac{E^{2}\left\{X_{k}\right\}}{2 \operatorname{var}\left\{X_{k}\right\}}\left[1+\left(\frac{H_{k}}{h_{k}}\right)^{2}\right]$, where $E\{$. and $\operatorname{var}\{$.$\} denote the expectation and variance, respectively and$ $\bar{x}_{k}=E\left\{X_{k}\right\}$ [25]. The parameters $H_{k}$ and $h_{k}$ may be defined in two unique ways that correspond to two distinct fading formats, where the difference arises from the physical interpretation of the parameter $\eta_{k}[10]^{4}$. In format $1,0<\eta_{k}<\infty$ is the power ratio of the in-phase and quadrature phase components of the fading signal in each multipath cluster, while $H_{k}$ and $h_{k}$ are given by:

$$
H_{k}=\frac{\eta_{k}^{-1}-\eta_{k}}{4} \text { and } h_{k}=\frac{2+\eta_{k}^{-1}+\eta_{k}}{4} .
$$

Moreover, in format 1 , the $\eta-\mu$ power distribution is symmetrical around $\eta_{k}=1$. The second format can be obtained from the first one using the relationship of $\eta_{\text {format } 2}=\frac{1-\eta_{\text {format } 1}}{1+\eta_{\text {format } 1}}[10]$.

On the other hand, the $\kappa-\mu$ shadowed power pdf is expressed as follows for each $X_{k}$ [11]:

$$
\begin{aligned}
f_{X_{k}, \kappa_{k}-\mu_{k} s h}(x)= & \frac{\mu_{k}^{\mu_{k}} m_{k}^{m_{k}}\left(1+\kappa_{k}\right)^{\mu_{k}} x^{\mu_{k}-1}}{\Gamma\left(\mu_{k}\right)\left(\bar{x}_{k}\right)^{\mu_{k}}\left(\mu_{k} \kappa_{k}+m_{k}\right)^{m_{k}}} \\
& \times e^{-\frac{\mu_{k}\left(1+\kappa_{k}\right) x}{\bar{x}_{k}}} 1 F_{1}\left(m_{k}, \mu_{k}, \frac{\mu_{k}^{2} \kappa_{k}\left(1+\kappa_{k}\right)}{\mu_{k} \kappa_{k}+m_{k}} \frac{x}{\bar{x}_{k}}\right),
\end{aligned}
$$

where $\kappa_{k}>0$ denotes the ratio of the total power of the dominant components to that of the scattered waves and $m_{k}$ is the shadowing parmeter. In (5), $\mu_{k}=\frac{E^{2}\left\{X_{k}\right\}}{\operatorname{var}\left\{X_{k}\right\}} \frac{1+2 \kappa_{k}}{\left(1+\kappa_{k}\right)^{2}}$ and $\bar{x}=$ $E\left\{X_{k}\right\}$, while ${ }_{1} F_{1}$ is the Kummer Hypergeometric function.

The elements $a_{k}$ for $1 \leq k \leq N$ have two important characteristics, which are as follows [4]:

1) Similarity: For a particular distribution model, the coefficients $a_{k}$ may or may not be identically distributed. Specifically, for the cases of the $\eta-\mu$ and $\kappa-\mu$ shadowed distributions, they may or may not all have the same $\left\{\eta_{k}, \mu_{k}\right\}$ and $\left\{\kappa_{k}, \mu_{k}, m_{k}\right\}$ parameters, respectively.

\footnotetext{
${ }^{4}$ It is important to note that the $\eta-\mu$ pdf well models the small-scale variations of the fading signal in a scenario of non-line-of-sight communication [10].
}

2) Correlation: For a particular distribution model, the coef- 218 ficients $a_{k}$ associated with $1 \leq k \leq N$ may or may not 219 be correlated with each other. The level of correlation is 220 represented by the correlation matrix as follows:

$$
\mathbf{C}_{\mathbf{m}}=\left[\begin{array}{cccccc}
\rho_{11} & \rho_{12} & \cdots & \rho_{1 j} & \cdots & \rho_{1 N} \\
\vdots & \vdots & \vdots & \rho_{i j} & \vdots & \vdots \\
\rho_{N 1} & \rho_{N 2} & \cdots & \rho_{1 j} & \cdots & \rho_{N N}
\end{array}\right],
$$

where $\rho_{i j}$ denote the correlation coefficient between $a_{i} 222$ and $a_{j}$. Note that $\mathbf{C}_{\mathbf{m}}$ is an identity matrix for the case of 223 all fading magnitudes being independent.

In our study, we employ Maximal Ratio Combining 225 (MRC) [4] detection, because its performance closely matches 226 the performance of the optimal maximum-likelihood detec- 227 tion [4], while its complexity is much lower. Assuming perfect 228 channel estimation, the received symbol $y$ after MRC is as 229 follows:

$$
y=\frac{\mathbf{h}^{H} \hat{\mathbf{y}}}{\mathbf{h}^{H} \mathbf{h}} .
$$

\section{B. Error Vector Magnitude}

The error vector between the transmitted complex-valued 232 symbol $s(l)=s_{I}(l)+j \cdot s_{Q}(l)$ and the received symbol $y(l)=233$ $y_{I}(l)+j \cdot y_{Q}(l)$ is defined as $e(l)=y(l)-s(l)$. Fig. 1 shows 234 a vectorial representation of $e$ using the constellation diagram 235 of the communication system. The EVM of the communication 236 system is proportional to the root mean square value of the error 237 signal $e(l)$. In other words, if a total of $L$ symbols are trans- 238 mitted over the wireless channel, then the EVM of the SIMO 239 system described in Section II-A may be expressed as follows 240 [24]:

$$
E V M=\sqrt{\frac{\frac{1}{L} \sum_{l=1}^{L}|y(l)-s(l)|^{2}}{P_{o}}},
$$

where $P_{o}$ is the average symbol power. If $s(l) \in 242$ $\left\{S_{1}, S_{2}, \cdots S_{M}\right\}$, and if all symbols are equi-probable, 243 then $P_{o}$ may be expressed as:

$$
P_{o}=\frac{\sum_{m=1}^{M}\left|S_{m}\right|^{2}}{M} .
$$

\section{ANAlytical StUdy OF THE EVM FOR SIMO CHANNELS}

The EVM in an AWGN SISO channel has been formulated 247 as follows for the case of data-aided receivers [23]:

$$
\mathrm{EVM}=\sqrt{\frac{1}{S N R_{\text {SISO }}}} \text { when } L \rightarrow \infty,
$$

where $S N R_{S I S O}$ is the channel's signal-to-noise-ratio at the 249 single receive antenna, $L$ is the number of symbols transmit- 250 ted over the wireless channel and $M$ is the number of unique 251 wireless symbols in the modulation scheme. 




Fig. 1. Vector representation of the error between symbols $s$ and $y$.

In the SIMO scenario, if we assume that the average signal to noise ratio at each receive antenna is $\gamma_{i}=\gamma$, then the instantaneous equivalent signal-to-noise-ratio of the overall SIMO system is [4], [24]:

$$
\gamma_{i n s t}=\sum_{k=1}^{N} a_{k}^{2} \gamma=N \gamma \times \frac{1}{N} \sum_{k=1}^{N} a_{k}^{2}=S N R_{S I M O} \times Z,
$$

where $S N R_{S I M O}=N \gamma$ is the average equivalent signal-tonoise-ratio of the overall SIMO system, which represents the power gain of using a higher number of receivers ${ }^{5}$. On the other hand, in (11), $Z=\frac{1}{N} \sum_{k=1}^{N} a_{k}^{2}$ is the diversity gain, which converges to 1 as the number of antennas increases (assuming each of the channel gains is normalized to have unit variance) and it hence helps overcome fading [9, P. 72]. In our simulations, we compare the EVM obtained in a SIMO channel to that of the SISO channel. Our goal is to study the diversity gain obtained by employing multiple receive antennas and not the power gain. Hence, we compare the SIMO channel to an equivalent SISO AWGN channel having a signal-to-noiseratio of $S N R_{S I S O}=S N R_{S I M O}=N \gamma$ in order to ensure the same average received power in both scenearios. Note that the BER or EVM performance of the SIMO system may be better than that of a SISO AWGN channel having an $S N R_{S I S O}=\gamma$, but will always be worse than that of a SISO AWGN channel having an $S N R_{S I S O}=S N R_{S I M O}=N \gamma$.

Now, employing the instantaneous SNR in (10) we obtain the instantaneous $\operatorname{EVM}$ to be $\operatorname{EVM}(z)=\sqrt{\frac{1}{S N R_{S I M O} \times z}}$ for $L \rightarrow$ $\infty$, where $\operatorname{EVM}(z)$ is the instantaneous $\operatorname{EVM}$ for the scenario of the diversity gain $Z=z$. The average EVM is formulated by employing the definition in [24] where, the average EVM is calculated by averaging over all possible values of $z$ using the following expression:

$$
\operatorname{EVM}=\int_{0}^{\infty} \operatorname{EVM}(z) f_{Z}(z) \mathrm{d} z
$$

\footnotetext{
${ }^{5}$ We employ the notation $S N R_{S I M O}$ for distinguishing between the power gain and diversity gain obtained by employing multiple receive antennas.
}

where $f_{Z}(z)$ is the pdf of $Z$. Let us now derive the exact 282 closed-form expressions for the EVM in a SIMO channel, while 283 considering two fading scenarios, namely the $\eta-\mu$ and $\kappa-\mu 284$ shadowed fading channels.

\section{A. $\eta$ - $\mu$ Fading SIMO Channel}

286

In order to derive the EVM for $\eta-\mu$ fading, we first have to 287 derive the pdf of $Z=\sum_{k=1}^{N} X_{k}$, where we have $X_{k}=\frac{1}{N} a_{k}^{2} .288$ Thus, each $X_{k}$ has the pdf given in (3) with $\bar{x}_{k}=E\left\{X_{k}\right\}=\frac{1}{N} 289$ and the distribution parameters of $\left\{\eta_{k}, \mu_{k}\right\}$. The moment gen- 290 erating function (MGF) for $X_{k}$ has been derived in [26]. In 291 [27], it has been shown that the MGF of $X_{k}$ can be repre- 292 sented as the product of the MGFs of two gamma distributed 293 RVs (RVs), where both these gamma RVs have the same shape 294 parameter $\alpha_{2 k-1}=\alpha_{2 k}=\mu_{k}$, but different scale parameters 295 of $\theta_{2 k-1}=\frac{\bar{x}_{k}}{2 \mu_{k}\left(h_{k}+H_{k}\right)}$ and $\theta_{2 k}=\frac{\bar{x}_{k}}{2 \mu_{k}\left(h_{k}-H_{k}\right)}$. Using this rela- 296 tionship, as well as the studies in [27] and [28], we can state 297 that $X_{k}=P_{k}+Q_{k}$, such that $P_{k} \sim \mathcal{G}\left(\alpha_{2 k-1}, \theta_{2 k-1}\right)$, and $Q_{k} \sim 298$ $\mathcal{G}\left(\alpha_{2 k}, \theta_{2 k}\right)$. Note that $\mathcal{G}\left(\alpha_{2 k}, \theta_{2 k}\right)$ denote the gamma distribu- 299 tion with shape parameter $\alpha_{2 k}$ and scale parameter $\theta_{2 k}$. Thus, 300 the sum of $N \eta$ - $\mu \mathrm{RVs}$ may be alternatively expressed as the 301 sum of $L=2 N$ Gamma RVs, where the pdf of the sum of $L 302$ Gamma RVs has been derived in [29]. Now, as stated earlier, 303 the pdf of $Z=\sum_{k=1}^{N} X_{k}$ has been derived to be the following 304 using the pdf of the sum of $2 N$ Gamma RVs [29]:

$$
\begin{aligned}
& f_{Z, \eta-\mu}(z)=\frac{z^{i=1} \alpha_{i}-1}{\prod_{i=1}^{2 N}\left(\theta_{i}\right)^{\alpha_{i}} \Gamma\left(\sum_{i=1}^{2 N} \alpha_{i}\right)} \\
& \Phi_{2}^{(2 N)}\left(\alpha_{1}, \cdots, \alpha_{2 N} ; \sum_{i=1}^{2 N} \alpha_{i} ; \frac{-z}{\theta_{1}}, \cdots, \frac{-z}{\theta_{2 N}}\right),
\end{aligned}
$$

where $\Phi_{2}^{(N)}$ (.) is the confluent Lauricella function [30]. We 306 can now substitute this $f_{Z, \eta-\mu}(z)$ into (12) for formulating the 307 average EVM.

1) EVM of $\eta-\mu$ SIMO Channel With Independent and 309 Nonidentically Distributed Branches:

Lemma 1: The EVM expression of the $\eta-\mu$ fading SIMO 311 channel having independent and non-identically distributed 312 (i.n.i.d) branches is given by

$$
\begin{gathered}
E V M_{\eta-\mu, i . n . i . d}=\frac{\sqrt{N \mu_{1}\left(1+\eta_{1}^{-1}\right)}}{\sqrt{S N R_{S I M O}}} \frac{\Gamma\left(2 \sum_{i=1}^{N} \mu_{i}-0.5\right)}{\Gamma\left(2 \sum_{i=1}^{N} \mu_{i}\right)} \\
\times F_{D}^{(2 N-1)}\left[0.5, \mu_{1}, \mu_{2}, \mu_{2}, \cdots, \mu_{N}, \mu_{N} ; 2 \sum_{i=1}^{N} \mu_{i} ;\right. \\
1-\frac{1}{\eta_{1}}, 1-\frac{\mu_{1}\left(1+\eta_{1}^{-1}\right)}{\mu_{2}\left(1+\eta_{2}^{-1}\right)}, 1-\frac{\mu_{1}\left(1+\eta_{1}^{-1}\right)}{\mu_{2}\left(1+\eta_{2}\right)} \cdots \\
\left.1-\frac{\mu_{1}\left(1+\eta_{1}^{-1}\right)}{\mu_{N}\left(1+\eta_{N}^{-1}\right)}, 1-\frac{\mu_{1}\left(1+\eta_{1}^{-1}\right)}{\mu_{N}\left(1+\eta_{N}\right)}\right]
\end{gathered}
$$

for $2 \sum_{i=1}^{N} \mu_{i}>0.5$ 
Proof: See Appendix I for the proof.

The expression of the EVM for the i.n.i.d case is given in terms of Lauricella's function of the fourth kind $F_{D}^{(N)}[$. [30]. The function $F_{D}^{(N)}\left[a, b_{1}, \cdots, b_{N} ; c ; x_{1}, \cdots, x_{N}\right]$ can be evaluated using the following single integral expression:

$$
\begin{aligned}
& \frac{\Gamma(c)}{\Gamma(a) \Gamma(c-a)} \int_{0}^{1} t^{a-1}(1-t)^{c-a-1} \prod_{i=1}^{N}\left(1-x_{i} t\right)^{-b_{i}} \mathrm{~d} t \\
& \text { where } \operatorname{Real}(c)>\operatorname{Real}(a)>0,
\end{aligned}
$$

where Real(.) returns the real part of the argument. Note that the condition $\operatorname{Real}(c)>\operatorname{Real}(a)>0$ is satisfied by Lauricella's function of the fourth kind $F_{D}^{(N)}$ [.], which appeared in (14).

Special Case 1: Now, we simplify the expression in (14) for the case of independent and identically distributed (i.i.d) fading SIMO channels. Substituting both $\eta_{i}=\eta$ and $\mu_{i}=\mu \forall i$ into (14) and then using the following identity:

$F_{D}^{(N)}\left[a, b_{1}, \cdots b_{n} ; c, x, \cdots, x\right]=2 F_{1}\left[a, b_{1}+\cdots+b_{N} ; c ; x\right]$,

where ${ }_{2} F_{1}[$.$] is the Gauss hypergeometric function [30], we$ obtain,

$$
\begin{aligned}
& \mathrm{EVM}_{\eta-\mu, i . i . d}=\frac{\sqrt{N \mu\left(1+\eta^{-1}\right)}}{\sqrt{S N R_{S I M O}}} \frac{\Gamma(2 N \mu-0.5)}{\Gamma(2 N \mu)} \\
& { }_{2} F_{1}\left[0.5, N \mu ; 2 N \mu ; 1-\frac{1}{\eta}\right] \text { for } 2 N \mu>0.5 .
\end{aligned}
$$

In the following, we show that the expression shown in (17) converges to the EVM expression of AWGN channel. Note that when fading parameters $\eta=1$ and $\mu$ tends to infinity, the $\eta-\mu$ channel should converge to an AWGN channel. By substituting $\eta=1$ and $\mu \rightarrow \infty$ in (17), it can be simplified to

$$
\begin{aligned}
\mathrm{EVM}_{A W G N} & =\lim _{\mu \rightarrow \infty} \frac{\sqrt{2 N \mu}}{\sqrt{S N R_{S I M O}}} \frac{\Gamma(2 N \mu-0.5)}{\Gamma(2 N \mu)} \\
& =\frac{1}{\sqrt{S N R_{S I M O}}} .
\end{aligned}
$$

This simplification follows from the fact that ${ }_{2} F_{1}[0.5, N \mu ; 2 N \mu ; 0]=1$. We now provide the upper bound of the EVM expression given in (17) so that the impact of fading parameter $\eta$ can be shown. Using the transformation ${ }_{2} F_{1}[a, b ; c ; z]=(1-z)_{2}^{c-a-b} F_{1}[c-a, c-b ; c ; z]$, we obtain:

$$
\begin{aligned}
& \mathrm{EVM}_{\eta-\mu, i . i . d}=\frac{\sqrt{N \mu\left(1+\eta^{-1}\right)}}{\sqrt{S N R_{S I M O}}} \frac{\Gamma(2 N \mu-0.5)}{\Gamma(2 N \mu)} \\
& { }_{2} F_{1}\left[2 N \mu-0.5, N \mu ; 2 N \mu ; 1-\frac{1}{\eta}\right]\left(\frac{1}{\eta}\right)^{\mu-0.5},
\end{aligned}
$$

340 and using the bound ${ }_{2} F_{1}\left[2 N \mu-0.5, N \mu ; 2 N \mu ; 1-\frac{1}{\eta}\right]<2$ $341 F_{1}\left[2 N \mu, N \mu ; 2 N \mu ; 1-\frac{1}{\eta}\right]\left(\frac{1}{\eta}\right)^{-\mu}$, the expression given in
(19) can be upper bounded as:

$$
\begin{aligned}
\mathrm{EVM}_{\eta-\mu, i . i . d} & <\frac{\sqrt{N \mu\left(1+\eta^{-1}\right)}}{\sqrt{S N R_{S I M O}}} \frac{\Gamma(2 N \mu-0.5)}{\Gamma(2 N \mu)}\left(\frac{1}{\eta}\right)^{-0.5} \\
& =\frac{\sqrt{N \mu(1+\eta)}}{\sqrt{S N R_{S I M O}}} \frac{\Gamma(2 N \mu-0.5)}{\Gamma(2 N \mu)}
\end{aligned}
$$

Hence, it is apparent from (20) that as $\eta$ increases, the EVM 343 increases. Recall that $\eta$ is the scattered-wave power ratio 344 between the in-phase and quadrature components of each clus- 345 ter of multipath and hence the EVM will be minimum when the 346 power of the in-phase and the quadrature components is equal. 347

Special Case 2: The Nakagami-m fading is a special case 348 of the $\eta-\mu$ fading associated with $\eta=1$ and $2 \mu=m^{\prime}$. Note 349 that $m^{\prime}$ is the shape parameter of the Nakagami- $m$ fading. 350 Substituting $\eta=1$ and $2 \mu=m$ in (14) and (17), we obtain 351 the following expressions for the i.n.i.d and i.i.d scenarios, 352 respectively:

$\mathrm{EVM}_{n, i . n . i . d}=\frac{\sqrt{N m_{1}^{\prime}}}{\sqrt{S N R_{S I M O}}} \frac{\Gamma\left(\sum_{i=1}^{N} m_{i}^{\prime}-0.5\right)}{\Gamma\left(\sum_{i=1}^{N} m_{i}^{\prime}\right)} \times$

$F_{D}^{(N-1)}\left[0.5, m_{2}^{\prime}, \cdots, m_{N}^{\prime} ; \sum_{i=1}^{N} m_{i}^{\prime} ; 1-\frac{m_{1}^{\prime}}{m_{2}^{\prime}}, \cdots, 1-\frac{m_{1}^{\prime}}{m_{N}^{\prime}}\right]$

for $\sum_{i=1}^{N} m_{i}^{\prime}>0.5$ and

$\mathrm{EVM}_{n, i . i . d}=\frac{\sqrt{N m^{\prime}}}{\sqrt{S N R_{S I M O}}} \frac{\Gamma\left(N m^{\prime}-0.5\right)}{\Gamma\left(N m^{\prime}\right)}$ for $N m^{\prime}>0.5$.

Using the following identity from [31]:

$$
\begin{aligned}
& \frac{\Gamma(n+a)}{\Gamma(n+b)}=n^{a-b}\left(1+\frac{(a-b)(a+b-1)}{2 n}+O\left(|n|^{-2}\right)\right) \\
& \quad \text { for large } n,
\end{aligned}
$$

the EVM of the i.i.d Nakagami-m scenario can be further 355 simplified to

$$
\begin{gathered}
\mathrm{EVM}_{n, \text { i.i.d }}=\frac{1}{\sqrt{S N R_{S I M O}}}\left(1+\frac{0.75}{2 N m^{\prime}}+O\left(\left|N m^{\prime}\right|^{-2}\right)\right) \\
\text { for large } N m^{\prime} .
\end{gathered}
$$

Note that the first term in (24) represents the EVM of an 357 AWGN channel, while the remaining terms in (24) represent 358 the contribution of the fading. We know that as the parameter $m 359$ decreases, the impact of fading becomes more severe, which is 360 confirmed by (24). A second point that may be noted from (24) 361 is that the EVM approaches that of an AWGN channel, when 362 the number of receive antennas tends to infinity and/or when 363 the fading parameter tends to infinity. 364

2) EVM of $\eta-\mu$ SIMO Channel With Correlated and 365 Identically Distributed Branches: $\quad 366$

Lemma 2: The EVM expression of a correlated $\eta-\mu$ SIMO 367 channel associated with an MRC-based receiver is given by: 368 


$$
\begin{aligned}
& \mathrm{EVM}_{\eta-\mu, c o r r}=\frac{1}{\sqrt{\hat{\theta}_{1} S N R_{S I M O}}} \frac{\Gamma\left(2 N \mu_{c}-0.5\right)}{\Gamma\left(2 N \mu_{c}\right)} \\
& \quad \times F_{D}^{(2 N-1)}\left(0.5, \mu_{c}, \cdots, \mu_{c} ; 2 N \mu_{c} ; 1-\frac{\hat{\theta}_{2}}{\hat{\theta}_{1}}, \cdots 1-\frac{\hat{\theta}_{2 N}}{\hat{\theta}_{1}}\right) \\
& \quad \text { for } 2 N \mu_{c}>0.5 .
\end{aligned}
$$

Proof: See Appendix II for the proof.

\section{B. $\kappa-\mu$ Shadowed Fading SIMO Channel}

In order to derive the EVM of a $\kappa-\mu$ shadowed faded channel, we first have to derive the pdf of $Z=\sum_{k=1}^{N} X_{k}$, where $X_{k}=$ $\frac{1}{N} a_{k}^{2}$. Thus, each $X_{k}$ has the pdf given in (5) with $\bar{x}_{k}=\frac{1}{N}$ and distribution parameters of $\left\{\kappa_{k}, \mu_{k}, m_{k}\right\}$. The pdf of $Z$ has been shown in [11] to be as follows:

$$
\begin{aligned}
& f_{Z, \kappa-\mu s h}(z)=\prod_{i=1}^{N} \frac{\left.\mu_{i}^{\mu_{i}} m_{i}^{m_{i}}\left(1+\kappa_{i}\right)^{\mu_{i}} z^{i=1}{ }^{N} \mu_{i=1}^{N} \mu_{i}\right)\left(\mu_{i} \kappa_{i}+m_{i}\right)^{m_{i}} \bar{x}_{i}^{\mu_{i}}}{\Phi_{2}^{(N)}\left(\mu_{1}-m_{1}, \cdots \mu_{N}-m_{N}, m_{1} \cdots m_{n} ; \sum_{i=1}^{N} \mu_{i} ;\right.} \\
& -\frac{\mu_{1}\left(1+\kappa_{1}\right) z}{\bar{x}_{1}}, \cdots,-\frac{\mu_{N}\left(1+\kappa_{N}\right) z}{\bar{x}_{N}},-\frac{\mu_{1} m_{1}\left(1+\kappa_{1}\right) z}{\left(\mu_{1} \kappa_{1}+m_{1}\right) \bar{x}_{1}} \\
& \left.\cdots-\frac{\mu_{N} m_{N}\left(1+\kappa_{N}\right) z}{\left(\mu_{N} \kappa_{N}+m_{N}\right) \bar{x}_{N}}\right) .
\end{aligned}
$$

We can now substitute $f_{Z, \kappa-\mu s h}(z)$ in (12) to obtain the average EVM.

1) EVM of $\kappa-\mu$ Shadowed Fading SIMO Channel With i.n.i.d Branches:

Lemma 3: The EVM expression of $\kappa-\mu$ shadowed fading SIMO channel having i.n.i.d branches is given by

$$
\begin{aligned}
& E V M_{\kappa-\mu s h, i . n . i . d}=\frac{\sqrt{N \mu_{1}\left(1+\kappa_{1}\right)}}{\sqrt{S N R_{S I M O}}} \frac{\Gamma\left(\sum_{i=1}^{N} \mu_{i}-0.5\right)}{\Gamma\left(\sum_{i=1}^{N} \mu_{i}\right)} \\
& F_{D}^{(2 N-1)}\left(0.5, \mu_{2}-m_{2}, \cdots, \mu_{2}-m_{2}, m_{1}, \cdots m_{N} ;\right. \\
& \sum_{i=1}^{N} \mu_{i} ; 1-\frac{\mu_{1}\left(1+\kappa_{1}\right)}{\mu_{2}\left(1+\kappa_{2}\right)}, \cdots, 1-\frac{\mu_{1}\left(1+\kappa_{1}\right)}{\mu_{N}\left(1+\kappa_{N}\right)}, \\
& \left.1-\frac{\left(\mu_{1} \kappa_{1}+m_{1}\right)}{m_{1}} \cdots 1-\frac{\left(\mu_{N} \kappa_{N}+m_{N}\right) \mu_{1}\left(1+\kappa_{1}\right)}{m_{N} \mu_{N}\left(1+\kappa_{N}\right)}\right), \\
& \text { for } \sum_{i=1}^{N} \mu_{i}>0.5 .
\end{aligned}
$$

Proof: See Appendix III for the proof.

Special Case 1: Now, we simplify the expression in (27) for the i.i.d scenario, where we set $\mu_{i}=\mu$ and $\kappa_{i}=\kappa \forall i$ to obtain:

$$
\begin{aligned}
& \mathrm{EVM}_{\kappa-\mu s h, i . i . d}=\frac{\sqrt{N \mu(1+\kappa)}}{\sqrt{S N R_{S I M O}}} \frac{\Gamma(N \mu-0.5)}{\Gamma(N \mu)} \\
& { }_{2} F_{1}\left[0.5, N m ;, N \mu ;-\frac{\mu \kappa}{m}\right] \text { for } N \mu>0.5 .
\end{aligned}
$$

In the following, we will show that the above expression con- 386 verges to the EVM expression of AWGN channel. Note that 387 when fading parameters $\kappa=0$ and $\mu$ tends to infinity, the $\kappa$ - 388 $\mu$ channel should converge to an AWGN channel. By putting 389 $\kappa=0$ and $\mu \rightarrow \infty$ the above expression can be simplified to 390

$$
\begin{aligned}
\mathrm{EVM}_{A W G N} & =\lim _{\mu \rightarrow \infty} \frac{\sqrt{N \mu}}{\sqrt{S N R_{S I M O}}} \frac{\Gamma(N \mu-0.5)}{\Gamma(N \mu)} \\
& =\frac{1}{\sqrt{S N R_{S I M O}}} .
\end{aligned}
$$

The above simplification follows from the fact that 391 ${ }_{2} F_{1}[0.5, N \mu ; 2 N \mu ; 0]=1$. Note that the EVM expres- 392 sion for an $\kappa-\mu$ shadowed channel converges to the expression 393 of AWGN channel when fading parameter $\kappa=0$ and $\mu$ tends 394 to infinity, as expected.

Special Case 2: We now derive the closed-form expression 396 of EVM for the $\kappa-\mu$ fading SIMO channel having i.i.d branches. 397 Note that the $\kappa-\mu$ fading is a special case of the $\kappa-\mu$ shadowed 398 fading with $m \rightarrow \infty$. Using the following identity [32]:

$$
\lim _{b \rightarrow \infty}{ }_{2} F_{1}\left[a, b, c, \frac{z}{b}\right]={ }_{1} F_{1}[a, c, z],
$$

the ${ }_{2} F_{1}[$.$] given in (28) can be simplified for m \rightarrow \infty$ as 400 follows:

$$
{ }_{2} F_{1}\left[0.5, N m ;, N \mu ;,-\frac{\mu \kappa}{m}\right]=1 F_{1}[0.5 ; N \mu ;-N \mu \kappa],
$$

where ${ }_{1} F_{1}[$.$] is the Kummer hypergeometric function [30]. 402$ Therefore, the EVM expression of the $\kappa-\mu$ fading SIMO chan- 403 nel having i.i.d branches is given by

$$
\begin{aligned}
& \mathrm{EVM}_{\kappa-\mu, i . i . d}=\frac{\sqrt{N \mu(1+\kappa)}}{\sqrt{S N R_{S I M O}}} \frac{\Gamma(N \mu-0.5)}{\Gamma(N \mu)} \\
& { }_{1} F_{1}(0.5, N \mu,-N \mu \kappa) \text { for } N \mu>0.5 .
\end{aligned}
$$

The EVM expression of the $\kappa-\mu$ fading SISO channel is 405 given by

$$
\begin{gathered}
\mathrm{EVM}_{\kappa-\mu}=\frac{\sqrt{\mu(1+\kappa)}}{\sqrt{S N R}} \frac{\Gamma(\mu-0.5)}{\Gamma(\mu)} 1 F_{1}(0.5, \mu,-\mu \kappa) \\
\text { for } \mu>0.5 .
\end{gathered}
$$

Additional validation of Equation (33): In the following, we 407 derive the EVM expression of the $\kappa-\mu$ fading SISO channel 408 using the negative moment given in [10] in order to fur- 409 ther validate our derivations ${ }^{6}$. The EVM for $\kappa-\mu$ channel is 410

\footnotetext{
${ }^{6}$ Note that the negative moment of sum of generalized fading distribution is not available and hence we cannot derive the EVM expression for SIMO channel.
} 
given by

$$
\mathrm{EVM}_{\kappa-\mu}=\int_{a=0}^{\infty} \sqrt{\frac{1}{a^{2} \mathrm{SNR}}} f_{\kappa-\mu}(a) \mathrm{d} a
$$

where $f_{\kappa-\mu}(a)$ is the $\kappa-\mu$ envelope probability density function. It is apparent from the above expression that the $\mathrm{EVM}_{\kappa-\mu}$ is the negative moment of the $\kappa-\mu$ fading distribution. Using the moment expression given in [10], the $\mathrm{EVM}_{\kappa-\mu}$ can be obtained as

$$
\begin{aligned}
& \operatorname{EVM}_{\kappa-\mu}=\frac{\sqrt{\mu(1+\kappa)}}{\sqrt{S N R}} \frac{\Gamma(\mu-0.5) \exp (-\kappa \mu)}{\Gamma(\mu)} \\
& { }_{1} F_{1}(\mu-0.5, \mu, \mu \kappa) \text { for } \mu>0.5 .
\end{aligned}
$$

Then, using the transformation $e_{1}^{-z} F_{1}(b-a, b, z)$ $=1 F_{1}(a, b,-z)$, we can simplify the above expression to

$$
\mathrm{EVM}_{\kappa-\mu}=\frac{\sqrt{\mu(1+\kappa)}}{\sqrt{S N R}} \frac{\Gamma(\mu-0.5)}{\Gamma(\mu)} 1 F_{1}(0.5, \mu,-\mu \kappa) .
$$

Therefore, we have shown that the expressions given in (35) and (33) are same. Note that the functional form of the pdf of the sum of correlated $\kappa-\mu$ shadowed random variables is similar to that of the sum of correlated $\eta-\mu$ random variables. Hence, the EVM expression for a correlated $\kappa-\mu$ shadowed SIMO channel can be derived in a similar manner to that of the $\eta-\mu$ SIMO channel. Furthermore, $\kappa-\mu$ fading is a special case of $\kappa-\mu$ shadowed fading and hence the EVM can be obtained numerically by employing a very high value of $m$ in the EVM expression for a $\kappa-\mu$ shadowed fading channel.

\section{Simulation Results}

In order to validate the EVM expressions derived for $\eta$ $\mu$ and $\kappa-\mu$ shadowed fading channels associated with the arbitary parameters, we simulated a BPSK modulation-based system communicating over these channels. We implemented a simulation-based solution of (12) using 1 transmit and $N$ receive antennas. The simulations employed the Monte Carlo approach, which relies on transmitting a large number of bits over the wireless channel and computing the average EVM. The simulations were carried out in Matlab.

Fig. 2 shows the EVM variation with respect to $S N R_{S I M O}$ for the case of SIMO channels having independent and nonidentically distributed branches, where it can be seen that the simulation results closely match the theoretical values.

Fig. 3 depicts the variation of EVM with respect to $S N R_{S I M O}$ for $\eta$ - $\mu$ fading. Here, we have considered $N=3$ and $\eta \geq 1$, since $\eta$ is symmetrical about 1 . Firstly, it may be seen that the analytical results match with the simulation results for the entire range of $S N R_{S I M O}$. Secondly, it may be observed that as $\eta$ increases, the EVM also increases for a fixed value of $\mu$. Recall that $\eta$ is the power ratio of the in-phase and quadrature-phase components of the fading signal in each multipath cluster. Hence, as the power ratio of the in-phase and

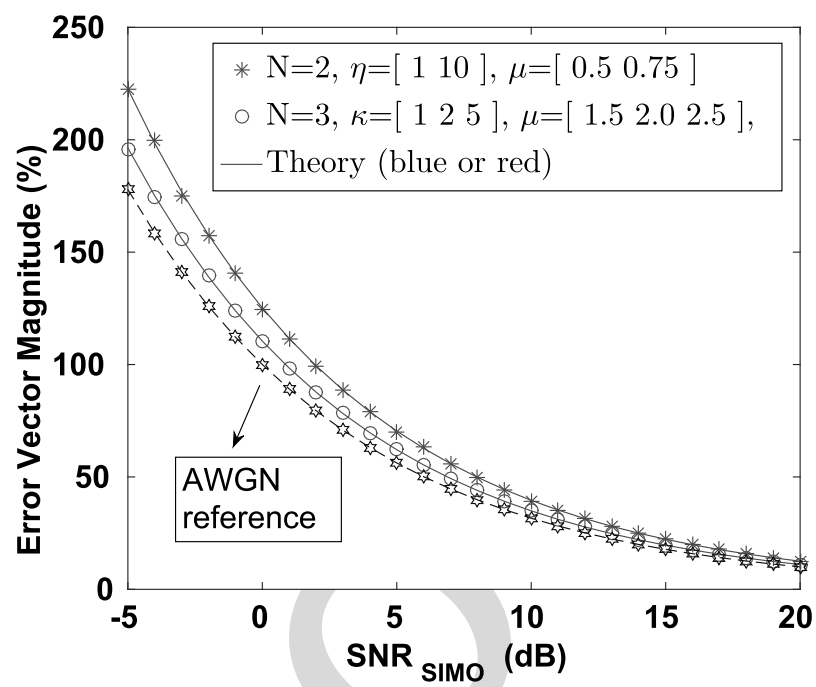

Fig. 2. The EVM for $\eta-\mu$ and $\kappa-\mu$ shadowed i.n.i.d SIMO channels.

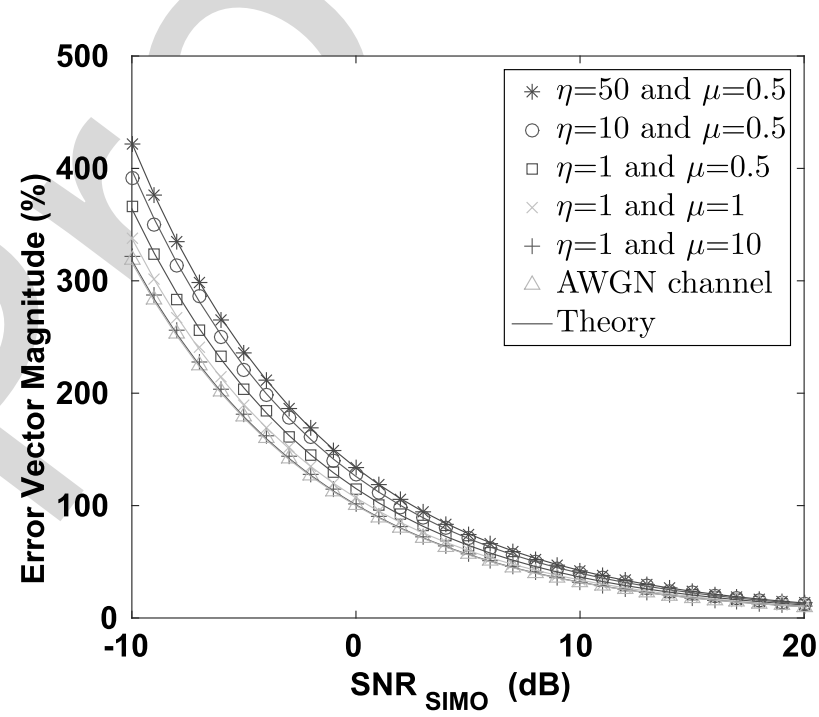

Fig. 3. The EVM for different values of $\eta$ and $\mu$, when $N=3$ and the channels are i.i.d.

quadrature-phase components increases, the EVM increases. In 453 other words, the EVM would be minimum, when the power 454 of the in-phase and quadrature-phase components of the fading 455 signal in each multipath cluster is equal. Thirdly, as the shape 456 parameter $\mu$ increases, the EVM decreases and it approaches 457 the EVM of an AWGN channel.

Fig. 4 shows the variation of EVM with respect to SNR $R_{S I M O} 459$ for different values of $N$. Firstly, observe that the simula- 460 tion results closely match the analytical results. Secondly, as 461 the number of antennas increases, the EVM decreases and it 462 approaches the EVM of an AWGN channel. Interestingly, it 463 may be seen that the EVM decreases significantly as the number 464 of antennas increases from 1 to 2 . However, the EVM reduction 465 becomes less significant, as the number of antennas increases 466 from $N=2$ to 3 and so on.

Fig. 5 shows the variation of EVM as a function of 468 $S N R_{S I M O}$ for different values of correlation coefficients. The 469 correlation between the SIMO branches is defined by the corre- 470 lation matrix in (6) in conjunction with $\rho_{p q}=\rho^{|p-q|}$, where 471 


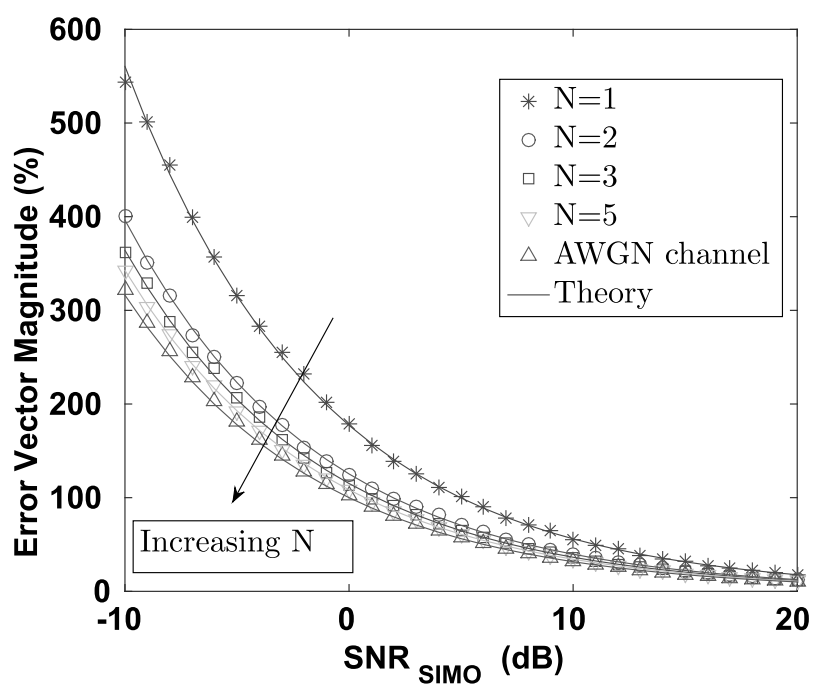

Fig. 4. The EVM for different values of $\mathrm{N}$, when $\eta=1$ and $\mu=0.5$ and the channels are i.i.d.

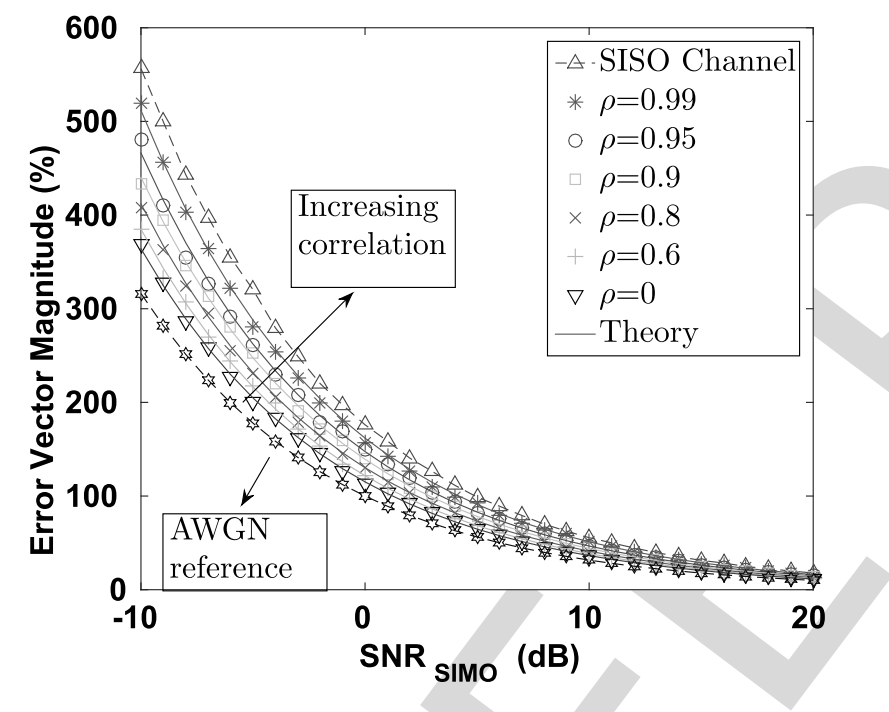

Fig. 5. The EVM for different values of correlation, when $N=3$, while $\eta=1$ and $\mu=0.5$ for all the channels. we have $p, q=1, \cdots, N$. Firstly, it may be seen that the simulation results closely match the analytical results for all values of the correlation coefficients. Secondly, it is observed that the EVM increases, as the correlation among the branches increases and it approaches the EVM of a SISO channel. Furthermore, the rate at which the EVM increases is higher, when the correlation coefficients are high.

Fig. 6 shows the variation of EVM versus the $N \times m$ product for the special case of Nakagami channels. It may be seen that the EVM decreases, as either $N$ or $m$ increases. Interestingly, the rate at which the EVM decreases is higher, when the number of antennas and the Nakagami-m fading parameter are small. This phenomenon may also be observed from (24), where the EVM of the Nakagami-m fading is shown to be a function of $1 /(N \times m)$.

Fig. 7 shows the EVM variation versus $S N R_{S I M O}$ for $\kappa-\mu$ fading. Again, the simulation results closely match the analytical results. It may be seen that as $\kappa$ increases, the EVM

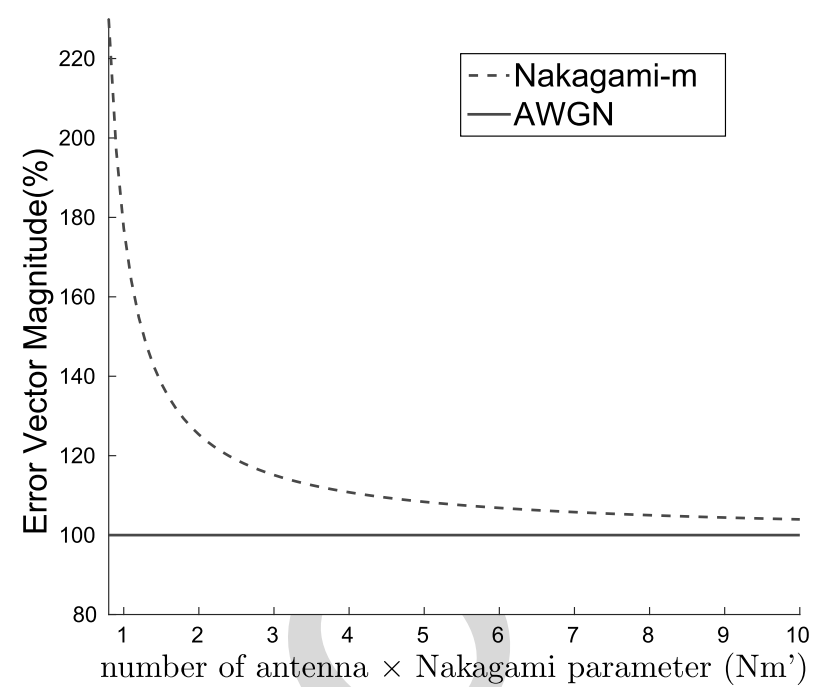

Fig. 6. Variation in EVM with respect to $N \times m^{\prime}$ for a Nakagami SIMO channel which are i.i.d.

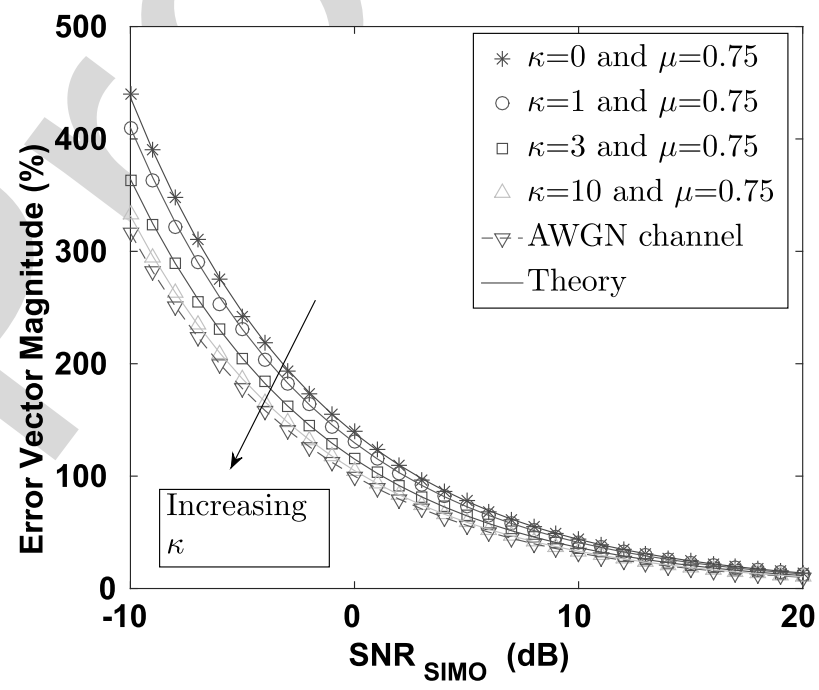

Fig. 7. The EVM for different combinations of $\kappa$ and $\mu$, when $N=2$ and the channels are i.i.d.

decreases and it approaches the EVM of an AWGN channel. 490 Recall that $\kappa$ represents the ratio of the total power of the dom- 491 inant components to that of the scattered waves. Hence, as the 492 ratio of the total power of the dominant components to that of 493 the scattered waves increases, the EVM decreases, as expected. 494

\section{Conclusions}

We have derived exact closed-form expressions for the data- 496 aided EVM in $\eta-\mu$ and $\kappa-\mu$ shadow faded SIMO channels 497 having independent and non-identically distributed branches. 498 The EVM expression is also derived for the scenario of cor- 499 related SIMO branches. Furthermore, the expressions derived 500 may be readily simplified for various special cases, such as 501 independent and identically distributed fading, the Rayleigh, 502 the Nakagami-m and finally the $\kappa-\mu$ fading. Subsequently, we 503 performed a simulation based study of this system in order to 504 validate the analytical results. Finally, a parametric study of the 505 
EVM performance of the wireless communication system considered showed that as the Nakagami fading parameter $m$ and/or the number of antennas $N$ increases, the EVM decreases and the rate at which the EVM decreases is higher, when the fading parameter and/or the number of antennas is small.

\section{ACKNOWLEDGMENT}

We are grateful to Dr. R. A. Shafik for his valuable inputs.

\section{APPENDIX A}

The EVM for a AWGN SISO channel is given by (10) [23], [24]. Thus, the instantaneous EVM, namely $E V M(z)$, is computed using (10) but with the replacement of $S N R_{S I S O}$ with the instantaneous signal-to-noise ratio, where $z S N R_{S I M O}$ is the instantaneous signal-to-noise ratio as per (11). Thus, the EVM of a $\eta-\mu$ fading channel is defined as follows [24]:

$$
\operatorname{EVM}_{\eta-\mu, i . n . i . d}=\int_{0}^{\infty} \operatorname{EVM}(z) f_{Z, \eta-\mu}(z) \mathrm{d} z
$$

which simply weights the AWGN channel's EVM by the specific probability of occurance of each particular instantaneous SNR given by its distribution and then averages it by integrating it across the entire instantaneous SNR range. Now substituting (13) in (36), we get:

$$
\begin{aligned}
& \operatorname{EVM}_{\eta-\mu, i . n . i . d}=\int_{0}^{\infty} \sqrt{\frac{1}{z S N R_{S I M O}}} \frac{z^{\sum_{i=1}^{2 N} \alpha_{i}-1}}{\prod_{i=1}^{2 N}\left(\theta_{i}\right)^{\alpha_{i}} \Gamma\left(\sum_{i=1}^{2 N} \alpha_{i}\right)} . \\
& \Phi_{2}^{(2 N)}\left(\alpha_{1}, \cdots, \alpha_{2 N} ; \sum_{i=1}^{2 N} \alpha_{i} ; \frac{-z}{\theta_{1}}, \cdots, \frac{-z}{\theta_{2 N}}\right) \mathrm{d} z \\
& =\int_{0}^{\infty} \sqrt{\frac{1}{S N R_{S I M O}}} \frac{\sum_{i=1}^{2 N} \alpha_{i}-1.5}{\prod_{i=1}^{2 N}\left(\theta_{i}\right)^{\alpha_{i}} \Gamma\left(\sum_{i=1}^{2 N} \alpha_{i}\right)} \\
& \Phi_{2}^{(2 N)}\left(\alpha_{1}, \cdots, \alpha_{2 N} ; \sum_{i=1}^{2 N} \alpha_{i} ; \frac{-z}{\theta_{1}}, \cdots, \frac{-z}{\theta_{2 N}}\right) \mathrm{d} z .
\end{aligned}
$$

Using the transformation [30, P. 177]:

$$
\begin{aligned}
& e^{-x_{i}} \Phi_{2}^{(n)}\left(b_{1}, \cdots, b_{n} ; c ; x_{1}, \cdots, x_{n}\right) \\
& \quad=\Phi_{2}^{(n)}\left(b_{1}, \cdot, b_{i-1}, c-b_{1}-\cdot-b_{n}, b_{i+1}, \cdot, b_{n} ; c ;\right. \\
& \left.\quad x_{1}-x_{i}, \cdots x_{i-1}-x_{i},-x_{i}, x_{i+1}-x_{i}, \cdot, x_{n}-x_{i}\right),
\end{aligned}
$$

the $\mathrm{EVM}_{\eta-\mu, \text { i.n.i.d }}$ can be rewritten as:

$$
\begin{aligned}
& \operatorname{EVM}_{\eta-\mu, i . n . i . d}=K_{1} \int_{0}^{\infty} z^{i=1} \alpha_{i}^{2 N} \alpha^{-\frac{z}{\theta_{1}}} \\
& \quad \times \Phi_{2}^{(2 N)}\left(0, \alpha_{2}, \cdots, \alpha_{2 N} ; \sum_{i=1}^{2 N} \alpha_{i} ; \frac{z}{\theta_{1}},\left(\frac{1}{\theta_{1}}-\frac{1}{\theta_{2}}\right) z\right. \\
& \left.\cdots\left(\frac{1}{\theta_{1}}-\frac{1}{\theta_{N}}\right) z\right) \mathrm{d} z
\end{aligned}
$$

where we have $K_{1}=\sqrt{\frac{1}{S N R_{S I M O}}} \frac{1}{\prod_{i=1}^{2 N}\left(\theta_{i}\right)^{\alpha_{i}} \Gamma\left(\sum_{i=1}^{2 N} \alpha_{i}\right)}$. Note that if 527 one of the numerator parameters of the series expansion of 528 $\Phi_{2}^{(N)}$ goes to zero, then $\Phi_{2}^{(N)}$ becomes $\Phi_{2}^{(N-1)}$ and hence the 529 above $\Phi_{2}^{(2 N)}$ will become $\Phi_{2}^{(2 N-1)}$ with appropriate parameters. 530 Using the transformation $\frac{z}{\theta_{1}}=t$ we obtain:

$$
\begin{aligned}
& \operatorname{EVM}_{\eta-\mu, i . n . i . d}=K_{1} \theta_{1}^{\sum_{i=1}^{2 N} \mu_{i}-0.5} \int_{0}^{\infty} \sum^{2 N} \alpha_{i=1}^{2 N} e^{-t} \\
& \times \Phi_{2}^{(2 N-1)}\left(\alpha_{2}, \cdots, \alpha_{2 N} ; \sum_{i=1}^{2 N} \alpha_{i} ;\left(1-\frac{\theta_{1}}{\theta_{2}}\right) t,\right. \\
& \left.\cdots\left(1-\frac{\theta_{1}}{\theta_{N}}\right) t\right) \mathrm{d} t .
\end{aligned}
$$

Using the following identity [30, P. 51]:

$$
\begin{aligned}
& F_{D}^{(n)}\left[a, b_{1}, \cdots, b_{n}, c, x_{1}, \cdots, x_{n}\right] \\
& =\frac{1}{\Gamma(a)} \int_{t=0}^{\infty} e^{-t} t^{a-1} \Phi_{2}^{(n)}\left[b_{1}, \cdots, b_{n}, c, x_{1} t, \cdots, x_{n} t\right] \mathrm{d} t
\end{aligned}
$$

where $\operatorname{Real}(a)>0$, one obtains:

$$
\begin{aligned}
& \mathrm{EVM}_{\eta-\mu, i . n . i . d}=K_{1} \theta_{1}^{\sum_{i=1}^{2 N} \alpha_{i}-0.5} \Gamma\left(\sum_{i=1}^{2 N} \alpha_{i}-0.5\right) \\
& \times F_{D}^{(2 N-1)}\left(\sum_{i=1}^{2 N} \alpha_{i}-0.5, \alpha_{2}, \cdots, \alpha_{2 N} ; \sum_{i=1}^{2 N} \alpha_{i}\right. \\
& \left.1-\frac{\theta_{1}}{\theta_{2}}, \cdots, 1-\frac{\theta_{1}}{\theta_{N}}\right)
\end{aligned}
$$

for $\sum_{i=1}^{2 N} \alpha_{i}>0.5$. Here $F_{D}^{(N)}\left[a, b_{1}, \cdots, b_{N} ; c ; x_{1}, \cdots, x_{N}\right]$ is 534 the Lauricella's function of the fourth kind. Again, using the 535 following identity:

$$
\begin{aligned}
& F_{D}^{(n)}\left[a, b_{1}, \cdots, b_{n}, c, x_{1}, \cdots, x_{n}\right]=\prod_{i=1}^{n}\left(1-x_{i}\right)^{-b_{i}} \\
& F_{D}^{(n)}\left[a, b_{1}, \cdots, b_{n}, c, \frac{x_{1}}{x_{1}-1}, \cdots, \frac{x_{n}}{x_{n}-1}\right]
\end{aligned}
$$

we arrive at:

$$
\begin{aligned}
& \operatorname{EVM}_{\eta-\mu, i . n . i . d}=K_{1} \theta_{1}^{\sum_{i=1}^{2 N} \alpha_{i}-0.5} \Gamma\left(\sum_{i=1}^{2 N} \alpha_{i}-0.5\right) \prod_{i=2}^{2 N}\left(\frac{\theta_{1}}{\theta_{i}}\right)^{-\alpha_{i}} \\
& \times F_{D}^{(2 N-1)}\left(0.5, \alpha_{2}, \cdots, \alpha_{2 N} ; \sum_{i=1}^{2 N} \alpha_{i} ; 1-\frac{\theta_{2}}{\theta_{1}}, \cdots, 1-\frac{\theta_{2 N}}{\theta_{1}}\right) .
\end{aligned}
$$

Substituting the value of $K_{1}$, the $\mathrm{EVM}_{\eta-\mu, i . n . i . d}$ expression can 538 


$$
\begin{aligned}
& \text { be simplified to } \\
& \operatorname{EVM}_{\eta-\mu, \text { i.n.i.d }}=\frac{1}{\sqrt{\theta_{1} S N R_{S I M O}}} \frac{\Gamma\left(\sum_{i=1}^{2 N} \alpha_{i}-0.5\right)}{\Gamma\left(\sum_{i=1}^{2 N} \alpha_{i}\right)} \\
& \times F_{D}^{(2 N-1)}\left(0.5, \alpha_{2}, \cdots, \alpha_{2 N} ; \sum_{i=1}^{2 N} \alpha_{i} ; 1-\frac{\theta_{2}}{\theta_{1}}, \cdots, 1-\frac{\theta_{2 N}}{\theta_{1}}\right) .
\end{aligned}
$$

Substituting the value of $\theta_{i}, \alpha_{i}$ and $\bar{z}=1 / N$ into (45), we obtain the final expression of $\mathrm{EVM}_{\eta-\mu, \text { i.n.i.d }}$ given in (14).

\section{APPENDIX B}

The underlying philosophy in this derivation is similar to that of an $\eta-\mu$ SIMO channel with i.n.i.d branches. We now consider the scenario studies in this paper, where $Z$ is the sum of $N$ correlated and identically distributed $\eta-\mu \mathrm{RVs} X_{k}$ having distribution parameters $\left\{\eta_{k}, \mu_{c}\right\}$. Note that all the $X_{k}$ s have the same $\mu_{c}$ but different $\eta_{k}$. As discussed in the first paragraph of Section III-A, an $\eta-\mu$ random variable may be expressed as the sum of two independent Gamma distributed RVs. It has been discussed in [28] that each $X_{k}$ may be expressed as

$$
X_{k}=P_{k}+Q_{k},
$$

where $P_{k} \sim \mathcal{G}\left(\mu_{c}, \theta_{2 k-1}\right)$, and $Q_{k} \sim \mathcal{G}\left(\mu_{c}, \theta_{2 k}\right)$ with $\theta_{2 k-1}=$ $\frac{\bar{x}_{k}}{2 \mu_{c}\left(h_{k}+H_{k}\right)}$ and $\theta_{2 k}=\frac{\bar{x}_{k}}{2 \mu_{c}\left(h_{k}-H_{k}\right)}$. Similar to Section III-A, $\bar{x}_{k}=1 / N$, while $h_{k}$ and $H_{k}$ are given by (4). Note that the correlation among the different $X_{k}$ s results in a correlation among the different $P_{k}$ s and among the $Q_{k}$ s, but there is no correlation between the $P_{k}$ s and $Q_{k}$ s. If $\rho_{i j}^{x x}$ is the correlation between $X_{i}=P_{i}+Q_{i}$ and $X_{j}=P_{j}+Q_{j}$, while $\rho_{i j}^{p p}$ and $\rho_{i j}^{q q}$ is the correlation between $\left\{P_{i}, P_{j}\right\}$ and $\left\{Q_{i}, Q_{j}\right\}$, respectively then we have (47), shown at the bottom of the page.

In our study $Z$ is the sum of $N$ correlated and identically distributed $\eta-\mu$ RVs $X_{k}$. Employing (46), we may state that $Z$ is the sum of $2 N$ correlated and non-identically distributed Gamma distributed RVs $M_{i}$, namely $\left\{M_{1}=P_{1}, M_{2}=\right.$ $\left.Q_{1}, M_{3}=P_{2}, M_{4}=Q_{2} \ldots, M_{2 N-1}=P_{N}, M_{2 N}=Q_{N}\right\}$.

The pdf of the sum of $N$ correlated $\eta-\mu$ math RVs is given by [16], [29], [33]

$$
\begin{aligned}
& F_{Z, \eta-\mu, c o r r}(z)=\frac{z^{2 N \mu_{c}-1}}{\operatorname{det}(\mathbf{A})^{\mu_{c} \Gamma\left(2 N \mu_{c}\right)}} \\
& \quad \times \Phi_{2}^{(2 N)}\left(\mu_{c}, \cdots, \mu_{c} ; 2 N \mu_{c} ; \frac{-z}{\hat{\theta}_{1}}, \cdots, \frac{-z}{\hat{\theta}_{2 N}}\right),
\end{aligned}
$$

where $\hat{\theta}_{i}$ is the eigen values of $\mathbf{A}=\mathbf{D C}$ with $\mathbf{D}$ being a diago- 568 nal matrix with entries $\theta_{i}$ and $\operatorname{det}(\mathbf{A})=\prod_{i=1}^{N} \hat{\theta}_{i}$ is the determinant 569 of the matrix A. Here, $\mathbf{C}$ is the symmetric positive definite 570 (s.p.d) matrix and is given in (49), shown at the bottom of the 571 page. where $\rho_{i j}^{m m}$ denotes the correlation coefficient between 572 $M_{i}$ and $M_{j}$, and is given by,

$$
\rho_{i j}^{m m}=\rho_{j i}^{m m}=\frac{\operatorname{cov}\left(M_{i}, M_{j}\right)}{\sqrt{\operatorname{var}\left(M_{i}\right) \operatorname{var}\left(M_{j}\right)}}, 0 \leq \rho_{i j} \leq 1,
$$

with $\operatorname{cov}\left(M_{i}, M_{j}\right)$ being the covariance between $M_{i}$ and $M_{j} .574$ Note that the alternate zeros are a consequence of $P_{k} \mathrm{~s}$ and $Q_{k} \mathrm{~s} \quad 575$ being independent.

Just as in (36), the EVM of a SIMO channel encountering 577 correlated $\eta-\mu$ fading and employing MRC reception is defined 578 as follows:

$$
\operatorname{EVM}_{\eta-\mu, c o r r}=\int_{0}^{\infty} \operatorname{EVM}(z) f_{Z, \eta-\mu, c o r r}(z) \mathrm{d} z,
$$

The functional form of the pdf of the sum of correlated gamma 580 $\mathrm{RVs}$ is similar to that of the sum of i.n.i.d. $\eta-\mu \mathrm{RVs}$, as given 581 in (13). Hence the EVM expression in (51) may be readily 582 simplified to:

$$
\begin{aligned}
& \mathrm{EVM}_{\eta-\mu, c o r r}=\frac{1}{\sqrt{\hat{\theta}_{1} S N R_{S I M O}}} \frac{\Gamma\left(2 N \mu_{c}-0.5\right)}{\Gamma\left(2 N \mu_{c}\right)} \\
& \times F_{D}^{(2 N-1)}\left(0.5, \mu_{c}, \cdots, \mu_{c} ; 2 N \mu_{c} ;\left(1-\frac{\hat{\theta}_{2}}{\hat{\theta}_{1}}\right), \cdots\left(1-\frac{\hat{\theta}_{2 N}}{\hat{\theta}_{1}}\right)\right)
\end{aligned}
$$

Note that $\hat{\theta}_{i}$ is the eigen values of $\mathbf{A}=\mathbf{D C}$ with $\mathbf{D}$ being a 584 diagonal matrix with entries $\theta_{i}$ and $\mathbf{C}$ is the symmetric positive 585 definite (s.p.d) covariance matrix defined in (49).

$$
\rho_{i j}^{x x}=\frac{\rho_{i j}^{p p} \sqrt{\operatorname{var}\left(P_{i}\right) \operatorname{var}\left(P_{j}\right)}+\rho_{i j}^{q q} \sqrt{\operatorname{var}\left(Q_{i}\right) \operatorname{var}\left(Q_{j}\right)}}{\sqrt{\operatorname{var}\left(P_{i}\right) \operatorname{var}\left(P_{j}\right)+\operatorname{var}\left(Q_{i}\right) \operatorname{var}\left(Q_{j}\right)+\operatorname{var}\left(P_{i}\right) \operatorname{var}\left(Q_{j}\right)+\operatorname{var}\left(P_{j}\right) \operatorname{var}\left(Q_{i}\right)}}
$$

$$
\mathbf{C}=\left[\begin{array}{ccccccccc}
1 & 0 & \sqrt{\rho_{12}^{p p}} & 0 & \sqrt{\rho_{13}^{p p}} & 0 & \cdots & \sqrt{\rho_{1 N}^{p p}} & 0 \\
0 & 1 & 0 & \sqrt{\rho_{12}^{q q}} & 0 & \sqrt{\rho_{13}^{q q}} & \cdots & 0 & \sqrt{\rho_{1 N}^{q q}} \\
\sqrt{\rho_{21}^{p p}} & 0 & 1 & 0 & \sqrt{\rho_{23}^{p p}} & 0 & \cdots & \sqrt{\rho_{2 N}^{p p}} & 0 \\
\vdots & \vdots & \vdots & \vdots & \vdots & \vdots & \vdots & \vdots & \vdots \\
0 & \sqrt{\rho_{N 1}^{q q}} & 0 & \sqrt{\rho_{N 2}^{q q}} & 0 & \sqrt{\rho_{N 3}^{q q}} & \cdots & 0 & 1
\end{array}\right] .
$$


$589 \beta_{i}=\frac{\bar{x}_{i}}{\mu_{i}\left(1+\kappa_{i}\right)}$ and $\delta_{i}=\frac{\left(\mu_{i} \kappa_{i}+m_{i}\right) \bar{x}_{i}}{\mu_{i}\left(1+\kappa_{i}\right) m_{i}}$, we can rewrite the pdf $590 f_{Z, \kappa-\mu s h}(z)$ as

$$
\begin{aligned}
& f_{Z, \kappa-\mu s h}(z)=\left(\prod_{i=1}^{N} \frac{1}{\beta_{i}^{\mu_{i}-m_{i}} \delta_{i}^{m_{i}}}\right) \frac{z^{\sum^{i=1}} \mu_{i}-1}{\Gamma\left(\sum_{i=1}^{N} \mu_{i}\right)} \\
& \times \Phi_{2}^{(2 N)}\left(\mu_{1}-m_{1}, \cdots, \mu_{N}-m_{N}, m_{1} \cdots, m_{N} ;\right. \\
& \left.\sum_{i=1}^{N} \mu_{i} ;-\frac{z}{\beta_{1}}, \cdots,-\frac{z}{\beta_{N}},-\frac{z}{\delta_{1}}, \cdots, \frac{z}{\delta_{N}}\right) .
\end{aligned}
$$

591 The EVM of $\kappa-\mu$ shadow fading SIMO channel with i.n.i.d 592 branches is defined as follows [24]:

$$
\operatorname{EVM}_{\kappa-\mu s h, i . n . i . d}=\int_{0}^{\infty} \operatorname{EVM}(z) f_{Z, \kappa-\mu s h}(z) \mathrm{d} z .
$$

593 Note that the functional form of the pdf of the sum of $\kappa-\mu$ shad-
594

595

596 owed RVs is similar to that of the sum of $\eta-\mu \mathrm{RVs}$, as given in (13). Hence the EVM of the $\kappa-\mu$ shadowed fading SIMO channel with i.n.i.d branches may be expressed as follows:

$$
\begin{aligned}
& \operatorname{EVM}_{\kappa-\mu s h, i . n . i . d}=\frac{1}{\sqrt{\beta_{1} S N R_{S I M O}}} \frac{\Gamma\left(\sum_{i=1}^{N} \mu_{i}-0.5\right)}{\Gamma\left(\sum_{i=1}^{N} \mu_{i}\right)} \\
& \times F_{D}^{(2 N-1)}\left(0.5, \mu_{2}-m_{2}, \cdots, \mu_{2}-m_{2}, m_{1}, \cdots m_{N} ;\right. \\
& \left.\sum_{i=1}^{N} \mu_{i} ; 1-\frac{\beta_{2}}{\beta_{1}}, \cdots, 1-\frac{\beta_{N}}{\beta_{1}}, 1-\frac{\delta_{1}}{\beta_{1}} \cdots 1-\frac{\delta_{N}}{\beta_{1}}\right) .
\end{aligned}
$$

Substituting the value of $\beta_{i}$ and $\delta_{i}$ and $\bar{x}_{i}=1 / N \forall i$ into (55), we obtain the final expression of $\mathrm{EVM}_{\kappa}-\mu$ sh,i.n.i.d, which is given in (27).

\section{REFERENCES}

[1] J. Zhang, Z. Tan, H. Wang, Q. Huang, and L. Hanzo, "The effective throughput of MISO systems over $\kappa-\mu$ fading channels," IEEE Trans. Veh. Technol., vol. 63, no. 2, pp. 943-947, Feb. 2014.

[2] V. Aalo, T. Piboongungon, and G. Efthymoglou, "Another look at the performance of MRC schemes in Nakagami-M fading channels with arbitrary parameters," IEEE Trans. Commun., vol. 53, no. 12, pp. 2002-2005, Dec. 2005.

[3] D. Chen, L.-L. Yang, and L. Hanzo, "Multi-hop diversity aided multi-hop communications: A cumulative distribution function aware approach," IEEE Trans. Commun., vol. 61, no. 11, pp. 4486-4499, Nov. 2013.

[4] L. Hanzo, S. X. Ng, T. Keller, and W. Webb, Quadrature Amplitude Modulation, 2nd ed. Hoboken, NJ, USA: Wiley, 2004.

[5] R. Vaughan, N. Scott, and D. White, "Eight hints for making and interpreting EVM measurements," Agilent Application Note, May 2005, pp. 1-12.

[6] R. Liu, Y. Li, H. Chen, and Z. Wang, "EVM estimation by analyzing transmitter imperfections mathematically and graphically," Analog Integr. Circuits Signal Process., vol. 48, no. 3, pp. 257-262, Jan. 2014.

[7] A. Georgiadis, "Gain, phase imbalance, and phase noise effects on error vector magnitude," IEEE Trans. Veh. Technol., vol. 53, no. 2, pp. 443449, Mar. 2004.
[8] R. Shafik, S. Rahman, R. Islam, and N. Ashraf, "On the error vector mag- 622 nitude as a performance metric and comparative analysis," in Proc. Int. 623 Conf. Emerg. Technol. (ICET), Nov 2006, pp. 27-31. 624

[9] D. Tse and P. Viswanath, Fundamentals of Wireless Communication. 625 Cambridge, U.K.: Cambridge Univ. Press, 2005.

[10] M. D. Yacoub, "The $\kappa-\mu$ distribution and the $\eta$ - $\mu$ distribution," IEEE 627 Antennas Propag. Mag., vol. 49, no. 1, pp. 68-81, Feb. 2007.

[11] J. Paris, "Statistical characterization of $\kappa-\mu$ shadowed fading," IEEE 629 Trans. Veh. Technol., vol. 63, no. 2, pp. 518-526, Feb. 2014.

[12] M. Arti, "Channel estimation and detection in hybrid satellite- 631 terrestrial communication systems," IEEE Trans. Veh. Technol., 2015, 632 http://ieeexplore.ieee.org/xpl/login.jsp?tp=\&arnumber=7156170, to be 633 published.

[13] M. Arti and M. Bhatnagar, "Beamforming and combining in hybrid 635 satellite-terrestrial cooperative systems," IEEE Commun. Lett., vol. 18, 636 no. 3, pp. 483-486, Mar. 2014.

[14] M. Arti and M. Bhatnagar, "Two-way mobile satellite relaying: A beam- 638 forming and combining based approach," IEEE Commun. Lett., vol. 18, 639 no. 7, pp. 1187-1190, Jul. 2014.

[15] M. Arti, "Imperfect CSI based maximal ratio combining in shadowed- 641 rician fading land mobile satellite channels," in Proc. 21st Nat. Conf. 642 Commun. (NCC), Feb. 2015, pp. 1-6.

[16] M. Bhatnagar, "On the sum of correlated squared $\kappa$ - $\mu$ shadowed ran- 644 dom variables and its application to performance analysis of MRC," IEEE 645 Trans. Veh. Technol., vol. 64, no. 6, pp. 2678-2684, Jun. 2015.

[17] J. F. Paris, "Outage probability in $\eta-\mu / \eta-\mu$ and $\kappa-\mu / \eta-\mu$ interference- 647 limited scenarios," IEEE Trans. Commun., vol. 61, no. 1, pp. 335-343, 648 Jan. 2013.

[18] D. da Costa and M. Yacoub, "Average channel capacity for generalized 650 fading scenarios," IEEE Commun. Lett., vol. 11, no. 12, pp. 949-951, 651 Dec. 2007.

[19] S. Kumar, G. Chandrasekaran, and S. Kalyani, "Analysis of outage prob- 653 vol. 19, no. 2, pp. 211-214, Feb. 2015.

[20] L. Hanzo, M. El-Hajjar, and O. Alamri, "Near-capacity wireless 6 transceivers and cooperative communications in the MIMO era: 657 Evolution of standards, waveform design, and future perspectives," Proc. 658 IEEE, vol. 99, no. 8, pp. 1343-1385, Aug. 2011. 659

[21] M. Bhatnagar and M. Arti, "On the closed-form performance analysis 660 of maximal ratio combining in shadowed-rician fading LMS channels," 661

[22] K. Gharaibeh, K. Gard, and M. Steer, "Accurate estimation of digital 663 communication system metrics-SNR, EVM and $\rho$; in a nonlinear ampli- 664 pp. 41-44.

[23] R. Shafik, S. Rahman, and R. Islam, "On the extended relationships 667 among EVM, BER and SNR as performance metrics," in Proc. Int. Conf. 668 Elect. Comput. Eng. (ICECE), Dec. 2006, pp. 408-411.

[24] H. Mahmoud and H. Arslan, "Error vector magnitude to SNR conver- 670 sion for nondata-aided receivers," IEEE Trans. Wireless Commun., vol. 8, 671 no. 5, pp. 2694-2704, May 2009.

[25] N. Ermolova and O. Tirkkonen, "Bivariate $\eta$ - $\mu$ fading distribution with 673 application to analysis of diversity systems," IEEE Trans. Wireless 674 Commun., vol. 10, no. 10, pp. 3158-3162, Oct. 2011.

[26] N. Y. Ermolova, "Moment generating functions of the generalized $\eta-\mu \quad 676$ and $\kappa-\mu$ distributions and their applications to performance evaluations of 677 communication systems," IEEE Commun. Lett., vol. 12, no. 7, pp. 502- 678 504, Jul. 2008.

[27] K. Peppas, F. Lazarakis, A. Alexandridis, and K. Dangakis "Error per- 680 formance of digital modulation schemes with MRC diversity reception 681 over $\eta$ - $\mu$ fading channels," IEEE Trans. Wireless Commun., vol. 8, no. 10, 682 pp. 4974-4980, Oct. 2009.

[28] N. Y. Ermolova and O. Tirkkonen, "Bivariate $\eta$ - $\mu$ fading distribution with application to analysis of diversity systems," IEEE Trans. Wireless Commun., vol. 10, no. 10, pp. 3158-3162, Oct. 2011.

[29] S. Kalyani and R. Karthik, "The asymptotic distribution of maxima of independent and identically distributed sums of correlated or nonidentical gamma random variables and its applications," IEEE Trans. Commun., vol. 60, no. 9, pp. 2747-2758, Sep. 2012.

[30] H. Exton, Multiple Hypergeometric Functions and Applications. New York, NY, USA: Halsted Press, Ellis Horwood, 1976.

[31] F. G. Tricomi and A. Erdlyi, "The asymptotic expansion of a ratio of 693 gamma functions," Pac. J. Math., vol. 1, no. 1, pp. 133-142, 1951.

32] N. M. Temme, "Large parameter cases of the gauss hypergeometric 695 function," J. Comput. Appl. Math., vol. 153, no. 1, pp. 441-462, 2003.696

[33] S. Kumar and S. Kalyani, "Coverage probability and rate for $\eta-\mu / \kappa-\mu \quad 697$ fading channels in interference-limited scenarios," IEEE Trans. Wireless 698 Commun., vol. 14, no. 11, pp. 6082-6096, Nov. 2015. ability and capacity for $\kappa-\mu / \eta-\mu$ faded channel," IEEE Commun. Lett., 654 IEEE Commun. Lett., vol. 18, no. 1, pp. 54-57, Jan. 2014. fier environment," in Proc. ARFTG Microw. Meas. Conf., Dec. 2004, 665

3
4
6
7
8
9
0
1
2
3
4
5
6
7
8
9
0
1
3
4
5
6
7
9

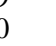

,

年

(1)

(1)

(6)

.

,




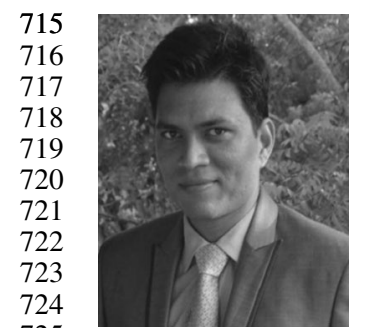

725

726

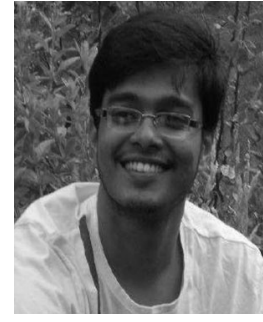
at Venice, Italy.
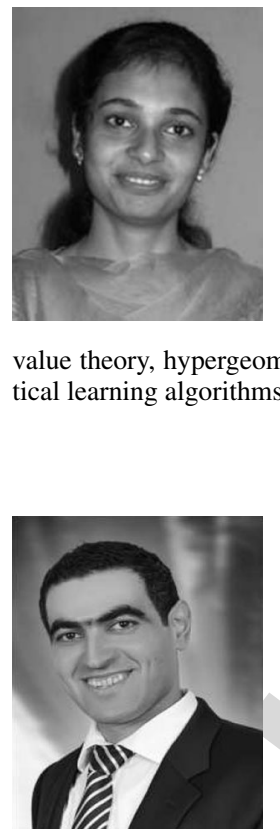

Varghese Antony Thomas received the B.E. (Hons.) degree in electrical and electronics engineering from Birla Institute of Technology and Science, Pilani (Goa Campus), India, in 2010, and the M.Sc. degree in wireless communications and the $\mathrm{Ph} . \mathrm{D}$. degree from the University of Southampton, Southampton, U.K., in 2011 and 2015, respectively. $\mathrm{He}$ is a Research Associate with Georgia Institute of Technology, Atlanta, GA, USA. As a Ph.D. student, he worked with the Wireless Communications Research Group of the University of Southampton. His research interests include optical communications, optical-wireless integration, backhaul for MIMO, and radio over fiber systems. He was the recipient of several academic awards including the Commonwealth Scholarship of the Government of U.K. and Mayflower Scholarship of University of Southampton.

Sheetal Kalyani received the B.E. degree in electronics and communication engineering from Sardar Patel University, Gujarat, India, in 2002, and the $\mathrm{Ph} . \mathrm{D}$. degree in electrical engineering from the Indian Institute of Technology (IIT) Madras, Chennai, India, in 2008. She was a Senior Research Engineer with the Centre of Excellence in Wireless Technology, Chennai, India, from 2008 to 2012. She is is currently an Assistant Professor with the Department of Electrical Engineering, IIT Madras.

Her research interests include HetNets, extreme value theory, hypergeometric functions, generalized fading models, and statistical learning algorithms for prediction in wireless networks.

Mohammed El-Hajjar received the B.Eng. degree in electrical engineering from the American University of Beirut, Lebanon, in 2004, and the M.Sc. degree in radio frequency communication systems and the Ph.D. degree in wireless communications from the University of Southampton, Southampton, U.K., in 2005 and 2008, respectively. Following the Ph.D., he joined Imagination Technologies as a Design Engineer, where he worked on designing and developing Imagination's multistandard communications platform, which resulted in three patents. Since January 2012, he has been a Lecturer with the Southampton Wireless Research Group, School of Electronics and Computer Science, University of Southampton, Southampton, U.K. He has authored a Wiley-IEEE book and more than 60 journal and international conference papers. His research interests include the development of intelligent communications systems including energy-efficient transceiver design, multifunctional MIMO, millimetre wave communications, and radio over fibre systems.

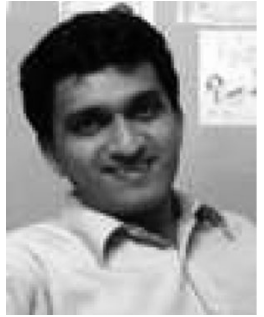

K. Giridhar received the B.Sc. degree in applied sci- 758 ences from PSG College of Technology, Coimbatore, 759 India, the M.E. degree in electrical communications 760 from Indian Institute of Science, Bangalore, India, 761 and the Ph.D. degree in electrical engineering from 762 the University of California, Santa Barbara, Santa 763 Barbara, CA, USA. Between 1989 and 1990, he 764 was a Member of Research Staff with CRL, Bharat 765 Electronics, Bangalore, India, and between 1993 and 766 1994, was a Research Affiliate in electrical engineer- 767 ing with Stanford University, Stanford, CA, USA. 768 Since 1994, he has been with the Department of Electrical Engineering, IIT 769 Madras (IITM), Chennai, India.

He serves as a Consultant to many Telecom \& VLSI companies in India, 771 and was on a Sabbatical from 2004 to 2005 with Beceem Communications. 772 He has been a Visiting Faculty at Sri Sathya Sai Institute of Higher Learning, 773 Prasanthi Nilayam, Anantapur, India, and Stanford University. His research 774 interests include adaptive signal processing and wireless communications sys- 775 tems, with an emphasis on various transceiver algorithms, custom air-interface 776 design for strategic applications, and performance analysis of mobile broadband 777 wireless networks including HetNets.

$\mathrm{He}$ is a Member of the Telecommunications and Computer Networks (TeNeT) Group at IITM. He actively collaborates with the Center of Excellence in Wireless Technology on MIMO-OFDM broadband access research, resulting in several contributions to IEEE $802.16 \mathrm{~m}$, and currently on proposals to LTE-A and $5 \mathrm{G}$ forums.$$
\text { and }
$$

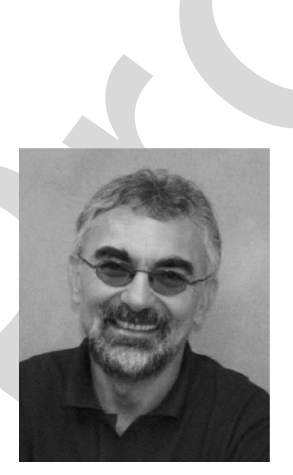

Lajos Hanzo (M'91-\&SM'92-F'04) received the degree in electronics in 1976 and the doctorate degree in 1983. During his 40-year career in telecommunications, he has held various research and academic posts in Hungary, Germany, and the U.K. Since 1986, he has been with the School of Electronics and Computer Science, University of Southampton, U.K., where he holds the chair in telecommunications. He has successfully supervised about $100 \mathrm{Ph} . \mathrm{D}$. students, co-authored 20 John Wiley/IEEE Press books on mobile radio communications totalling in excess of 10,000 pages, published over 1500 research entries at IEEE Xplore, acted both as the TPC and the General Chair of IEEE conferences, presented keynote lectures, and has been awarded a number of distinctions. Currently, he is directing a 60-strong academic research team, working on a range of research projects in the field of wireless multimedia communications sponsored by industry, the Engineering, and Physical Sciences Research Council (EPSRC), U.K., the European Research Council's Advanced Fellow Grant, and the Royal Society's Wolfson Research Merit Award. His research is funded by the European Research Council's Senior Research Fellow Grant. Lajos has over 23,000 citations. He is an enthusiastic supporter of industrial and academic liaison and offers a range of industrial courses. He is a Fellow of Royal Academy of 805 Engineering, the Institution of Engineering and Technology, the EURASIP, and 806 DSc. He is also a Governor of the IEEE VTS. From 2008 to 2012, he was the 807 Editor-in-Chief of the IEEE Press and also a Chaired Professor at Tsinghua 808 University, Beijing, China. In 2009, he was awarded an Honorary Doctorate 809 by the Technical University of Budapest, while in 2015 by the University of 810 Edinburgh.
784 785 786 787 , , , 